\title{
Aeroacoustic Simulations of a Nose Landing Gear using FUN3D on Pointwise Unstructured Grids
}

\author{
Veer N. Vatsa* \\ Mehdi R. Khorrami* \\ NASA Langley Research Center, Hampton, VA 23681 \\ John Rhoads ${ }^{\dagger}$ \\ Pointwise Inc., 213 S. Jennings Ave., Fort Worth, TX 76104 \\ and \\ David P. Lockard ${ }^{\ddagger}$ \\ NASA Langley Research Center, Hampton, VA 23681
}

\begin{abstract}
Numerical simulations have been performed for a partially-dressed, cavity-closed (PDCC) nose landing gear configuration that was tested in the University of Florida's open-jet acoustic facility known as the UFAFF. The unstructured-grid flow solver FUN3D is used to compute the unsteady flow field for this configuration. Mixedelement grids generated using the Pointwise ${ }^{\circledR}$ grid generation software are used for these simulations. Particular care is taken to ensure quality cells and proper resolution in critical areas of interest in an effort to minimize errors introduced by numerical artifacts. A hybrid Reynolds-averaged Navier-Stokes/large eddy simulation (RANS/LES) turbulence model is used for these simulations. Solutions are also presented for a wall function model coupled to the standard turbulence model. Time-averaged and instantaneous solutions obtained on these Pointwise grids are compared with the measured data and previous numerical solutions. The resulting CFD solutions are used as input to a Ffowcs Williams-Hawkings noise propagation code to compute the farfield noise levels in the flyover and sideline directions. The computed noise levels compare well with previous CFD solutions and experimental data.
\end{abstract}

\section{Nomenclature}

$C_{p} \quad\left(p-p_{\infty}\right) /\left(0.5 \rho_{\infty} u_{\infty}^{2}\right)$

$\mathrm{d} \quad$ distance normal to the wall (meter)

$\mathrm{dB} \quad$ decibels

TKE turbulence kinetic energy $\left(\right.$ meter $\left.^{2} / \mathrm{sec}^{2}\right),\left[\left(u_{r m s}^{\prime}\right)^{2}+\left(v_{r m s}^{\prime}\right)^{2}+\left(w_{r m s}^{\prime}\right)^{2}\right] / 2$

2-D TKE two-dimensional turbulence kinetic energy $\left(\operatorname{meter}^{2} / \mathrm{sec}^{2}\right),\left[\left(u_{r m s}^{\prime}\right)^{2}+\left(v_{r m s}^{\prime}\right)^{2}\right] / 2$

$u, v, w \quad$ Cartesian fluid velocity components (meter/sec)

$u_{\tau} \quad$ friction velocity (meter/sec)

$X, Y, Z \quad$ Cartesian coordinates

$y^{+} \quad$ distance normal to surface in wall units, $u_{\tau} d / \nu$

$\theta \quad$ circumferential angle, measured clockwise from wheel leading edge

$\rho \quad$ density $\left(\mathrm{kg} / \mathrm{meter}^{3}\right)$

$\mu \quad$ bulk viscosity ( $\mathrm{kg} /$ meter sec)

$\nu \quad$ kinematic viscosity, $\mu / \rho\left(\right.$ meter $\left.^{2} / \mathrm{sec}\right)$

*Aerospace Engineer, Computational AeroSciences Branch, Research Directorate; Associate Fellow AIAA

${ }^{\dagger}$ Senior Engineer; Senior Member AIAA

${ }^{\ddagger}$ Aerospace Engineer, Computational AeroSciences Branch, Research Directorate; Senior Member AIAA 


\begin{tabular}{|c|c|}
\hline \multicolumn{2}{|c|}{ Conventions } \\
\hline BART & Basic Aerodynamic Research Tunnel \\
\hline BDF2OPT & optimized second-order backward differencing formulation \\
\hline CFD & computational fluid dynamics \\
\hline DES & detached eddy simulation \\
\hline DDES & delayed detached eddy simulation \\
\hline FW-H & Ffowcs Williams-Hawkings \\
\hline LES & large eddy simulation \\
\hline MDDES & modified delayed detached eddy simulation \\
\hline NLG & nose landing gear \\
\hline PDCC & partially-dressed, cavity-closed \\
\hline PIV & particle image velocimetry \\
\hline PSD & power spectral density \\
\hline RANS & Reynolds-averaged Navier-Stokes \\
\hline SPL & sound pressure level \\
\hline SST & shear stress transport model \\
\hline UFAFF & University of Florida Aeroacoustic Flow Facility \\
\hline
\end{tabular}

Superscript

perturbation quantity (e.g., $u^{\prime}=u-u_{\infty}$ )

$\begin{array}{ll}\text { Subscript } & \\ \text { mean } & \text { time-averaged quantity } \\ \text { rms } & \text { root mean square } \\ \infty & \text { free-stream quantity }\end{array}$

\section{Introduction}

In recent years, there has been a growing emphasis on reducing airframe noise to meet the increasingly aggressive standards for permissible aircraft noise near airports. The landing gear is a significant contributor to airframe noise during approach and landing of commercial aircraft, ${ }^{1}$ and, therefore, understanding of the noise sources associated with such configurations is very important. Prediction of noise sources around the landing gear of an aircraft is an extremely challenging problem despite the progress made in the field of computational fluid dynamics (CFD) during the last several decades. The geometric complexity and highly chaotic unsteady flow fields associated with landing gear pose significant challenges for numerical simulation. In addition to the physical modeling difficulties associated with complex unsteady flows, generating suitable grids that can resolve boundary layer, shear layer and wake regions of interest for such configurations is an extremely demanding and time consuming task.

For the current work, we make use of the unstructured-grid flow code FUN3D, ${ }^{2}$ in which the compressible NavierStokes equations are solved to simulate the viscous unsteady flow field. The FUN3D code has been used for many large-scale applications. ${ }^{3}$ These include simulations of unsteady flow past single- and tandem-cylinder configurations, ${ }^{4}$ where time-averaged and perturbation quantities were shown to be in good agreement with experimental data and earlier computations from the well-established, structured-grid flow code CFL3D. ${ }^{5}$ The FUN3D code has also been used to compute the flow over landing gear configurations, and the resulting solutions on manually enriched unstructured grids were shown to compare well with experimental data. ${ }^{6,7}$ The grids used for the landing gear configuration in these earlier studies were generated with the widely-used VGRID code developed by Pirzadeh, ${ }^{8}$ and required significant human effort and time. As reported by Khorrami and Mineck, ${ }^{9}$ VGRID has also been used to generate grids for predicting the airframe noise of essentially a full aircraft configuration. Although the VGRID code has performed well in the past for a variety of applications, to the best of our knowledge, it is currently in maintenance mode with no further improvements planned. Hence, for the foreseeable future, there is no path for VGRID to overcome existing deficiencies or incorporate new features.

One of our objectives in this paper is to demonstrate the use of an alternate grid generation methodology for simulation of unsteady flow over complex configurations, such as a realistic landing gear configuration. After a careful examination of the resources and grid generation software available, the Pointwise grid generation code was selected for evaluation for such applications. In recent years, Pointwise, Inc. has developed a comprehensive software tool kit 
for generating hybrid (structured/unstructured and mixed-element) grids for complex geometries. Here, we make use of this software for generating prismatic grids near solid surfaces that transition to tetrahedral grids outside of boundary layer regions. Dense grids were created to resolve high gradients in the shear layer and wake regions associated with sharp edges and bluff bodies of the landing gear configuration. Based on our current experience, Pointwise offers a more up-to-date and efficient alternative to VGRID for generating grids that are suitable for aeroacoustic computations of such complex configurations.

\section{Governing Equations and Flow Solver}

In the present work, the spatially second-order accurate CFD solver FUN3D is used to obtain numerical solutions of the unsteady Navier-Stokes equations. A hybrid approach is used where the Reynolds-averaged Navier-Stokes (RANS) equations supplemented by turbulence models are solved in regions where the grid is inadequate to resolve the unsteady flow features, such as near solid walls, and a large eddy simulation (LES) approach is used in the remainder of the computational domain. The details of the blending between these regions and the subgrid scale model are dependent on the turbulence model used for simulations..$^{10,11}$

FUN3D is a suite of codes, including an unstructured-grid flow solver, adjoint solver, and grid adaption software. The FUN3D unstructured-grid flow solver, developed originally by Anderson and Bonhaus, ${ }^{12}$ has gone through significant modifications over the years by a team of NASA Langley Research Center researchers. ${ }^{13}$ The discrete form of the governing equations is solved in a time-accurate manner with a constant time-step at every grid point. At each iteration step, a linear system of equations is relaxed with a point implicit procedure. ${ }^{14}$ For the current work, Roe's flux-difference splitting scheme ${ }^{15}$ is used without a flux limiter.

A dual time-stepping algorithm ${ }^{16}$ with subiterations is employed in FUN3D to converge the solution within each time-step. For these simulations, 15 subiterations per time-step were used, which was adequate to obtain at least 3 orders of magnitude reduction in the residuals of the governing equations. An optimized second-order backward differencing formulation (BDF2OPT) scheme ${ }^{17}$ was chosen for the current work. This scheme produces higher temporal accuracy compared to the standard second-order backward differencing scheme at nominally the same computational cost but with slightly increased memory ( 6 additional 3D arrays) usage. The time-step was chosen so that approximately 20 points per wavelength are used to represent a $10 \mathrm{kHz}$ signal, which should be adequate to resolve unsteady signals up to frequencies of $10 \mathrm{kHz}$ with a second order temporal scheme.

The turbulence model under consideration here is based on the one-equation Detached Eddy Simulation (DES) model of Spalart. ${ }^{18}$ Although the DES model has been used often for solving unsteady separated flows, this model depends too strongly on the grid quality and topology, and can lead to non-physical results with grid refinement in viscous layers. Spalart et al. ${ }^{19}$ have subsequently modified the DES model to overcome some of the shortcomings related to its grid dependence resulting in a new version of the model named Delayed Detached Eddy Simulation or DDES. In the DDES model, a blending function that varies between 0 for RANS mode and 1 for LES mode is applied to the destruction terms of the turbulence model. A slight modification of this model was suggested by Vatsa and Lockard ${ }^{4}$ to overcome numerical difficulties associated with the non-physical behavior of the eddy-viscosity observed in the upstream region of cylindrical bluff bodies. In this modified model, the blending function suggested by Spalart et al. ${ }^{19}$ for the DDES model for the destruction terms is also applied to the production terms of the turbulence model, and it will be referred to as the modified DDES, or "MDDES" model, in this paper.

Carlson et al. ${ }^{20}$ have recently incorporated a wall function model in the FUN3D code. This model has been shown to work well with the one-equation Spalart-Allmaras model ${ }^{21}$ and the two-equation shear stress transport (SST) model of Menter $^{22}$ for a variety of aerodynamic problems. One of the nice features of this formulation is its applicability to grids where the near wall spacing can can vary from $y^{+}=O(1)$, which is typical of a viscous grid suitable for integration to the wall, to grids with wall spacing of $y^{+}=O(10-100)$. This is accomplished via a blending function patterned after the work of Knopp et al. ${ }^{23}$ The reader is referred to the original paper of Carlson et al. ${ }^{20}$ for further implementation details of this model. For the current applications, this wall function model is coupled with the Spalart-Allmaras based MDDES turbulence model for solving the unsteady flow equations.

\section{Simulated Configuration and Grid Generation Methodology}

The configuration under consideration here is the 1/4-scale, high-fidelity replica of a partially-dressed closed-cavity (PDCC) Gulfstream G550 nose landing gear that was tested by Zawodny et al. ${ }^{24}$ in the University of Florida's Aeroacoustic Flow Facility (UFAFF), an anechoic open-jet tunnel. In this PDCC configuration, several smaller components, such as the hydraulic and electric lines, the lighting cluster, and the steering assembly were removed from the fully- 
dressed flight model. Additionally, the door cavity, where the landing gear is stowed during cruise mode, was closed in this simplified model. PIV measurements for the same model were made in the BART tunnel at the NASA Langley Research Center to complement the acoustic measurements of the UFAFF facility.

A schematic of the computational domain chosen for current simulations of the nose landing gear configuration test setup in the UFAFF is shown in Fig. 1. In these simulations, the nose gear assembly is attached to a flat mounting plate, which is suspended in space. The computational domain enclosing the gear assembly and mounting plate is comprised of inflow, outflow, top, bottom and side boundaries as shown in Fig. 1. The mounting plate used in these simulations corresponds to the hardware used for supporting the landing gear assembly in the experimental work of Ref. 24.

The UFAFF is a large, anechoic facility, where the walls and collectors are treated to minimize the reflection of acoustic waves. It is impractical to replicate such details in numerical simulations. To reduce the reflections from the computational boundaries, outer boundaries of the computational domain used in the current simulations are placed further away from the landing gear model in comparison with the physical dimensions of the UFAFF test section. For example, the inflow boundary was placed further upstream of the landing gear compared to the nozzle exit location in the experimental setup. Outflow boundary was also placed downstream of the collector for the same reason. The current choice of computational domain is an idealization of the open jet UFAFF experimental setup, and follows the guidelines suggested for numerical simulations for this case in the BANC-III airframe noise workshop. ${ }^{25}$

The grids for this work were created using Pointwise ${ }^{\circledR}$ version 17.2R 2 and contained 62 million and 45 million node mixed-elements for the wall-resolved and wall function simulations, respectively. The nominal value of $y^{+}$for these grids are approximately 1 and 25. Thus, the 62 million node grid is well suited for standard viscous wall treatment, whereas the 45 million node grid is better suited for simulations utilizing wall-functions. The same surface mesh was used to generate both of these grids to eliminate potential effects of changes in surface resolution on the solution. Due to the dynamic nature of the processes responsible for acoustic production, fairly stringent constraints were placed on the computational mesh beyond those typically associated with steady, turbulent flows. Most notably, regions of flow separation must maintain a high density of points away from the body to reduce numerical diffusion. This is of particular importance in areas where the separated wake impinges on material surfaces downstream. Increased resolution (node count) will undoubtedly increase the computational cost for the solution. For this reason, targeting critical regions of interest and locally refining the grid provides the benefits of off-body resolution for improved numerical accuracy without undue increase in overall node count.

The wheel cavity for the landing gear was found to be particularly susceptible to diffusive effects arising from insufficient off-body resolution. Preliminary simulations performed on an initial grid, depicted in Fig. 2 (a) near the wheel cavity, resulted in poor fluctuating surface pressures at the coordinates corresponding to the Kulite ${ }^{\circledR}$ sensor located on the downstream side of the wheel cavity. Pointwise provides the ability to control local grid size in unstructured blocks through the use of baffles, which serve as internal virtual faces to an unstructured block. By placing a baffle matching the surface resolution that loosely encloses the wheel cavity, the cell size within the cavity is constrained, resulting in a finer grid density suitable for resolving the separated shear layer originating at the sidewall of the tire. The effect of the baffle can be seen in the slice shown in Fig. 2 (b), where the location of the baffle is denoted with a red line roughly coinciding with the time-averaged position of the shear layer. A similar process was used in other critical off-body high-gradient regions for producing high-quality dense cells to improve the accuracy of the numerical solutions.

Planar cuts at the torque arm and wheel midplane (at locations shown schematically in Fig. 3) from the 62 million node grid are compared in Figs. 4-5 with a 104 million node reference grid generated using VGRID. Details of the 104 million node grid generation process and resulting solutions were presented at the BANC-III workshop held in Atlanta on June 14-15, 2014. ${ }^{25}$ An exploded view of these grids in a small, focus region aft of the wheel midplane (marked on Fig. 5) are compared in Fig. 6.

Note that the 62 million node Pointwise grid is comparable to the 104 million reference grid in the proximity of solid surfaces and in near-wake regions, whereas the grid density is coarsened in low gradient regions further away from solid surfaces. This strategy results in significant savings in overall size of the grid without sacrificing solution accuracy. A close examination of the grid in the vicinity of the wheel indicates that the surface grid density for the 62 million node grid is actually finer compared to the reference 104 million node grid (Figs. 6 and 7). In addition, these figures clearly indicate that the 62 million node grid gets progressively coarser in regions away from solid surfaces, thus resulting in a reduced number of grid points. Compared to the 62 million node grid, the 45 million node grid has a much coarser near-wall normal spacing, resulting in reduced grid count without sacrificing the resolution in off-body shear layers and wake regions. 


\section{Results}

To match the experimental test conditions of Ref. 24, the computations were performed at a freestream Mach number of 0.166 and a Reynolds number of $7.3 \times 10^{4}$ based on the main strut (piston) diameter of 0.75 inches. The computational domain chosen for numerical solutions (see Fig. 1) represents the open-jet (no solid boundaries) test set up of the UFAFF tunnel. Since the experiments were conducted with transition strips to ensure turbulent separation, the computations were run in a fully-turbulent mode. A constant wall temperature based on flat plate adiabatic wall conditions and no-slip conditions were imposed on viscous surfaces, which included the gear, fuselage and mounting plate. At the inflow plane, total pressure and total temperature corresponding to the wind tunnel conditions were imposed. Farfield conditions based on Riemann invariants were imposed at the outflow, sides, top, and bottom boundaries. Detailed description of these boundary conditions is available in Ref. 26

The FUN3D flow solver was run first in a steady mode starting from freestream conditions to expedite the development of the overall flow-field in these simulations. The solver was then run in time-accurate (unsteady) mode for 40,000 time steps to purge the initial disturbances from the computational domain before performing time-averaging of flow quantities. The unsteady data for the aeroacoustic analysis was collected at every other time step during the final 97,500 time steps of the computations, which results in unsteady records of 0.48 second duration. A similar strategy was used for obtaining the solutions on the 45 million node grid.

The computed time-averaged surface pressure distribution on the port wheel for the 62 million node grid $\left(y^{+}=1\right)$ is plotted in Fig. 8 as a function of the circumferential angle $\theta$, which is measured in the clockwise direction from the wheel leading edge. These results are denoted by the legend "PW-62M $\left(y^{+}=1\right)$ " in these figures. Solutions obtained on the Pointwise 45 million node grid $\left(y^{+}=25\right)$ with the newly developed wall function capability in FUN3D ${ }^{20}$ are also shown in this figure, and denoted by the legend "PW-45M $\left(y^{+}=25\right)$ ". Finally, the solutions based on the 104 million node reference grid and the experimental data are also included in this figure for comparison. The 62 million node Pointwise grid solutions are found to be in excellent agreement with the experimental data and previous 104 million node grid solution. It is encouraging to note that the solution obtained with wall-functions in FUN3D on the 45 million node grid is very similar to the 62 million node solution except for slight deviations in localized regions near the $\theta=130$ and 230 degree locations on the wheel.

Instantaneous streamwise velocity contours along the torque-arm cut (see Figs. 3-4) from the Pointwise grid solutions are compared with the 104 million node grid solutions in Fig. 9. Although these results can not be used for quantitative comparisons due to different time evolution histories, these are still useful for qualitative comparisons. The appearance of the wakes and shear layers created by the upstream strut and the torque-arm on the 62 million and 45 million node grids are very similar to the ones from the 104 million node case, at least in the near wake and the component interaction zones. As suspected from the mesh comparison of Fig. 4, the far-wake region for the 62 and 45 million node grids is not as well resolved, but it is not expected to have a significant effect on perturbation pressures on the landing gear and the farfield noise. In general, the solutions on the 45 and 62 million node grids presented here are qualitatively very similar to the 104 million node grid reference solution in shear layers and near-wake regions.

The time-averaged streamwise velocity contours along the torque-arm cut are compared for these grids in Fig. 10. In addition, results from PIV measurements acquired in the BART tunnel for this configuration are also presented in this figure for comparison. The computed results on the Pointwise grids are in very good agreement with the 104 million node reference grid solution. Although the computed results indicate somewhat larger low velocity regions in the near wake compared to the PIV data, the overall shape and growth of the shear layer in the wake region is well predicted.

The two-dimensional turbulence kinetic energy (2-D TKE), $\left[\left(u_{r m s}^{\prime}\right)^{2}+\left(v_{r m s}^{\prime}\right)^{2}\right] / 2$, contours on these grids along the same planar cut are presented in Fig. 11. The overall levels and distribution of 2-D TKE on the 62 million node grid are very similar to the results on the 104 million node grid, both qualitatively and quantitatively, except for somewhat higher TKE levels that persist further downstream in the 104 million node grid case, which has finer grid resolution in the farwake region. The results obtained with wall functions on the 45 million node grid are also shown in this figure. Note that the 2-D TKE levels in the shear layer and wake regions on this grid are comparable to the ones obtained for the 62 million node grid. Compared to the experimental data, computations indicate much higher levels of 2-D TKE in the wake region. It is not clear if this is due to the possible errors in the second moments, that are more susceptible to measurement errors compared to mean quantities, or if it is due to turbulence modeling and other errors in computed results. It should also be pointed out that the experimental PIV data used here is from the closed wall BART tunnel, whereas, the computations simulated the open jet UFAFF tunnel. Unfortunately, PIV data from the UFAFF is not available at this time for such comparisons.

Similar comparisons for the time-averaged velocities and 2-D TKE are presented along a plane downstream of the mid-section of the wheels in Figs. 12 and 13, respectively. Once again, the solutions from Pointwise grids and the reference 104 million node grid are in excellent agreement with each other for the streamwise velocity. The major 
difference is a slight under-prediction of reverse flow velocities in the near-wake region for the 45 million node case compared to finer grid results. The overall agreement of computed results with the measured velocity data is quite good. The 2-D TKE distributions indicate high levels in the shear layers developing at the edges of wheels, which then decays further downstream. High concentration of 2-D TKE is also observed in the gap between the wheels, which most probably is caused by the high level of unsteady fluctuations in the wake region downstream of the strut and wheel axle. The 45 million node grid solution obtained with wall functions for the 2-D TKE compare well with the 62 million node grid solution and with the 104 million node solution. The overall agreement of the numerical solutions with the experimental data, including the maximum level and streamwise decay rate of 2-D TKE is quite good. The lower 2-D TKE levels for the Pointwise grids in the far-wake region compared to the 104 million node grid solutions are attributed to coarser Pointwise grids in that region, as seen in Figs. 5 (a)-(b).

\section{IV.A. Surface Pressure Power Spectral Density}

Next, we examine the surface pressure power spectral density (PSD) distributions, which are calculated from the time-history of the unsteady surface pressures. Numerical unsteady data was collected at several locations on the gear corresponding to the position of Kulite ${ }^{\circledR}$ pressure sensors used in the experimental studies. ${ }^{24,27}$ These Kulite ${ }^{\circledR}$ sensor locations are shown in Fig. 14 for reference. Because the PSD distributions are directly related to the unsteady surface pressures that are also used as input to noise propagation codes, it is important to obtain good solutions for surface PSD distributions as a prerequisite for accurate noise prediction.

The computed PSD distributions from this study are compared with the experimental data in Fig. 15 at two locations on the door corresponding to Kulite ${ }^{\circledR}$ pressure sensors (channels) 3 and 10, and at sensors 7 and 15 in Fig. 16. The computational results from the 104 million node grid are also plotted in these figures for reference. Based on these results, it is observed that the PSD distributions on the 45 and 62 million node grids are in very good agreement with each other, except for small differences at lower frequencies. Also, the agreement between these solutions and experimental data is comparable to the reference solutions on the 104 million node grid up to a frequency of $2 \mathrm{kHz}$. Note that the PSD levels beyond this frequency are dropping rapidly for the 104 million node reference grid. In contrast, the spectra from the 45 and 62 million node Pointwise grids are generally in much better agreement with the experimental data and display only minor drop-offs in PSD levels at the higher frequencies. It is well known that for a numerical scheme of given spatial and temporal order accuracy, the number of mesh points required for accurate representation of waves is directly proportional to the frequency of interest. The improved high-frequency behavior observed for the Pointwise grids in these figures is therefore attributed to finer surface and near-body resolution (see Fig. 7) of these grids compared to the 104 million node grid that was used in earlier studies.

\section{IV.B. Farfield Noise}

Zawodny et al. ${ }^{24}$ have conducted aeroacoustic testing in the UFAFF, an open jet anechoic tunnel of the PDCC Gulfstream G550 nose landing gear configuration that is analyzed in this paper. In this experiment, a series of 11 microphones were placed at a distance of approximately 7.5 wheel diameters below the wheels along the flyover direction as shown in Fig. 17 (a). Acoustic measurements were also made at 9 microphones placed to the side of this model (see Fig. 17 (b)) at a distance of 5.7 wheel diameters from the centerline. The measured spectra at the microphone locations were corrected to account for shear layer refraction.

Farfield noise was computed with a conventional hybrid CFD/Acoustic Analogy Approach, where the pressure fluctuations on the solid surfaces of the landing gear configuration obtained from the CFD solutions were used as input to the Ffowcs Williams-Hawkings ${ }^{28}$ (FW-H) solver PSU-WOPWOP ${ }^{29,30}$ for computing the 1/3 Octave sound pressure levels (SPL) in $\mathrm{dB}$ (decibels) at farfield points. The results obtained in this manner at flyover microphones 4, 7, and 9 from the 45 and 62 million node Pointwise grid simulations are shown in Figs. 18 (a)-(c), respectively. It is observed from these figures that the 45 million node grid spectra obtained with wall functions are within just a few decibels of those from the 62 million node grid levels for these microphone locations. In addition to these solutions on Pointwise grids, the results on the reference grid of 104 million nodes are also presented here for comparison. These FW-H computations include the contribution of all the pertinent solid surfaces, i.e., the gear, fuselage and mounting plate. The experimental data from the UFAFF, which represents the noise levels at these locations from all the sources for this configuration, is shown as solid black lines connecting filled circles. The numerical results presented here for the Pointwise grids are in reasonably good agreement with the 104 million node results up to a frequency of $2 \mathrm{kHz}$, after which the predicted noise level from 104 million node grid displays a sharp decrease compared to the 62 million node Pointwise grid and the experimental data. These trends are very similar to what was observed for the PSD distributions in Figs. 15 and 16. Keeping in mind the fact that the experimental data at very low frequencies may be corrupted by the facility background 
noise, the 62 million node numerical solutions presented here are in very good agreement with the measurements up to a frequency of $4 \mathrm{kHz}$. As mentioned in the previous section, finer surface and near-body resolution of Pointwise grids result in improved accuracy of surface pressures at higher frequencies. Since these surface pressure distributions are used as input for the farfield noise predictions, similar accuracy at higher frequencies is achieved in farfield noise levels for the Pointwise grids. The implication here is that one must pay significant attention to surface mesh generation for aeroacoustic applications before tackling the problem of generating the volumetric grids.

The farfield noise results for sideline microphones 3, 5, 7 and 9 are presented in Figs. 19 (a)-(d), respectively. The computational results obtained on the Pointwise 45 and 62 million node grids are compared with the solution on the 104 million node reference grid, and with the experimental data. Overall agreement between the solutions on these three different grids is quite good. The computed spectra from the Pointwise grids are in fairly good agreement with the experimental data up to a frequency of 3-4 kHz. The predicted noise levels decay quite rapidly beyond $4 \mathrm{kHz}$ for the Pointwise grids, and somewhat sooner for the 104 million node grid. Once again, the wall function results on the 45 million node grids indicate the usefulness and applicability of this approach for predicting farfield noise generated by complex landing gear configuration on coarser grids. Because there is very little overhead associated with the wallfunction implementation in FUN3D, the reduced node count associated with wall function grids results in significant savings in computational costs to obtain solutions of comparable accuracy for aeroacoustic problems of practical interest.

\section{IV.C. Beamform Maps}

Beamform (or source localization) maps can be used to further analyze the acoustic signals by identifying the location and relative strengths of prominent noise sources. Zawodny et al. ${ }^{24}$ acquired farfield noise measurements in the UFAFF with a phased array of 64 microphones that was placed along the flyover and sideline planes of the landing gear configurations. A top view of the flyover array is shown in Fig. 20 (a), whereas a front view of the sideline array is shown in Fig. 20 (b). The flyover array is placed approximately 8 wheel diameters below the wheels, whereas the sideline array is placed to the side of the model at a distance of 9 wheels diameters from the model centerline. These phased acoustic arrays are placed in a quiescent region outside the shear layers induced by the open jet of the UFAFF tunnel. The results from the beamform analysis are presented here along the flyover direction, which is considered more important from community noise perspective.

For the present analysis, pressure records at the phased array microphone locations were generated using the FW-H approach in conjunction with the computed surface pressures. The AVEC, Inc beamforming suite ${ }^{31}$ was used to analyze the synthetic acoustic array data. This included standard beamforming processing and the deconvolution based algorithm proposed by Sijtsma. ${ }^{32}$ A typical source localization (beamform) map reconstructed from the unsteady pressure fluctuations at the 64 locations corresponding to the flyover acoustic array of Ref. 24 is presented in Fig. 21 at a frequency of $1 \mathrm{kHz}$. Results from the Pointwise 62 million node grid are compared with those obtained on the 104 million node reference grid in this figure. The maps from both sets of computations are very similar, and identify the near wake region of the wheels as the biggest source of noise at this frequency. A slight asymmetry w.r.t. the model centerline is observed in these contour maps. Most probably, this is due to the unsteady shedding caused by the slight asymmetry of connecting rods (struts) and cavity door surface geometries in the gear assembly, and a lack of symmetry in the grids used here.

Similar plots at $3 \mathrm{kHz}$ and $5 \mathrm{kHz}$ frequencies are shown in Figs. 22 and 23, respectively. The computed noise sources on both grids compare well with each other and indicate a forward movement of the primary noise source as the frequency is increased from $1 \mathrm{kHz}$ to $3 \mathrm{kHz}$. In general, the results from the Pointwise 62 million node grid produce a higher sound pressure level (SPL) compared to the 104 million grid case, except at the lower end of the frequency range. This is consistent with the farfield noise levels shown in Fig. 19. At a frequency of $3 \mathrm{kHz}$, the primary source of noise is around the wheel axle for both of these grids, as seen in Fig. 22. In addition to this, a weaker noise source is visible near the cavity door at this frequency for both grids. At $5 \mathrm{kHz}$ (Fig. 23), the contour map from the Pointwise grid shows one main source around the axle, but the 104 million node reference case shows additional noise sources around the edges of the wheels and on the cavity door. However, the peak source strength for the 104 million node grid is $51.2 \mathrm{~dB}$ compared with $66 \mathrm{~dB}$ for the Pointwise 62 million node grid. These results indicate that the Pointwise grid with a finer surface mesh is supporting stronger unsteadiness around the axle, especially at the higher frequencies.

\section{Concluding Remarks}

Hybrid RANS/LES based unsteady solutions for turbulent viscous flow over a realistic nose landing gear configuration are presented here on mixed-element grids generated with the Pointwise grid generation code. This approach is found to be very robust and efficient for generating grids suitable for aeroacoustic simulations of complex configurations. Computed time-averaged pressure distributions on these grids are shown to be in good agreement with experimental data 
and previous solutions on a reference (larger) grid.

Surface PSD values extracted from the current unsteady flow solutions at several sensor locations are compared with previous solutions and experimental data. In general, the solution accuracy on the current 62 million node grid is found to be comparable, if not better, than the reference 104 million node grid. Similar observations are made for the farfield noise at selected microphone locations. The results obtained on the 45 million node Pointwise grid using wall functions are shown to compare well with the 62 million node grid solutions and the experimental data.

Farfield noise levels calculated from these computations at selected locations in flyover and sideline directions on the Pointwise grids are shown to compare well with the experimental data and the 104 million node reference grid solution. Based on the results obtained so far, the wall function approach appears to offer a more efficient means for aeroacoustic simulations of practical configurations. In general, the Pointwise grid solutions for the surface PSD and farfield noise levels are in better agreement with the measured data at higher frequencies compared to the previously published results on a larger, 104 million node grid. This is attributed to finer surface and near-body grid resolutions of the current Pointwise grids.

Preliminary results for beamform maps used to identify the most significant noise sources are also presented here. Work is continuing on conducting such analysis with other independent tools, and these results will be compared with the experimental data in the near future. The ultimate goal is to identify the important noise source regions, and subsequently interrogate the CFD data to identify flow features responsible for the noise. Identification of these features may suggest modifications to the gear geometry that could reduce the noise, which would be the focus for future work.

\section{Acknowledgments}

This work was supported by NASA's Fundamental Aerodynamics and Integrated Systems Research Programs through the Fixed-Wing and Environmentally Responsible Aviation projects.

\section{References}

\footnotetext{
${ }^{1}$ Khorrami, M. R., Lockard, D. P., Humphreys, Jr., W. M., Choudhari, M. M., and Van de Ven, T., "Preliminary Analysis of Acoustic Measurements from the NASA-Gulfstream Airframe Noise Flight Test," AIAA Paper 2008-2814, May 2008.

${ }^{2}$ Biedron, R. T., Derlaga, J. M., Gnoffo, P. A., Hammond, D. P., Jones, W. T., Kleb, B., Lee-Rausch, E. M., Nielsen, E. J., Park, M. A., Rumsey, C. L., Thomas, J. L., and Wood, W. A., "FUN3D Manual: 12.4,” NASA TM 2014-218179, March 2014.

${ }^{3}$ FUN3D Web page: http://fun3d.larc.nasa.gov, March 2014.

${ }^{4}$ Vatsa, V. and Lockard, D., "Assessment of Hybrid RANS/LES Turbulence Models for Aeroacoustics Applications," AIAA Paper 2010-4001, June 2010.

${ }^{5}$ Rumsey, C., Biedron, R., and Thomas, J., “CFL3D: Its History and Some Recent Applications,” NASA TM 112861, May 1997, presented at the Godonov's Method for Gas Dynamics Symposium, Ann Arbor, MI.

${ }^{6}$ Vatsa, V., Lockard, D., and Khorrami, M., "Application of FUN3D Solver for Aeroacoustics Simulation of a Nose Landing Gear Configuration," AIAA Paper 2011-2820, June 2011.

${ }^{7}$ Vatsa, V., Lockard, D., Khorrami, M., and Carlson, J.-R., "Aeroacoustic Simulation of a Nose Landing Gear in an Open Jet Facility using FUN3D," AIAA Paper 2012-2280, June 2012.

${ }^{8}$ Pirzadeh, S., "Three-dimensional Unstructured Viscous Grids by the Advancing Layer Method," AIAA Journal, Vol. 33, No. 1, 1996, pp. 43-49.

${ }^{9}$ Khorrami, M. R. and Mineck, R. E., “Toward Full Aircraft Airframe Noise Prediction: Detached Eddy Simulations,” AIAA Paper 2014-2480, June 2014.

${ }^{10}$ Lynch, C. and Smith, M. J., "Hybrid RANS-LES Turbulence Models on Unstructured Grids,” AIAA Paper 2008-3854, June 2008.

${ }^{11}$ Baurle, R. and Edwards, J., "Hybrid Reynolds-Averaged/Large-Eddy Simulations of a Coaxial Supersonic Free-Jet Experiment,” AIAA Paper 2009-0129, January 2009.

${ }^{12}$ Anderson, W. K. and Bonhaus, D. L., "An Implicit Upwind Algorithm for Computing Turbulent Flows on Unstructured Grids," Computers and Fluids, Vol. 23, No. 1, 1994, pp. 1-21.

${ }^{13}$ Alexandrov, N., Atkins, H. L., Bibb, K. L., Biedron, R. T., Gnoffo, P. A., Hammond, D. P., Jones, W. T., Kleb, W. L., Lee-Rausch, E. M., Nielsen, E. J., Park, M. A., Raman, V. V., Roberts, T. W., Thomas, J. L., Vatsa, V. N., Viken, S. A., White, J. A., and Wood, W. A., "Team Software Development for Aerothermodynamic and Aerodynamic Analysis and Design," NASA TM 2003-212421, Nov. 2003.

${ }^{14}$ Nielsen, E., Lu, J., Park, M., and Darmofal, D., "An Implicit, Exact Dual Adjoint Solution Method for Turbulent Flows on Unstructured Grids," Computers and Fluids, Vol. 33, No. 9, 2003, pp. 1131-1155.

${ }^{15}$ Roe, P. L., “Approximate Riemann Solvers, Parameter Vectors, and Difference Schemes," Journal of Computational Physics, Vol. 43, No. 2, 1981, pp. 357-372.

${ }^{16}$ Biedron, R., Vatsa, V., and Atkins, H., "Simulation of Unsteady Flows using an Unstructured Navier-Stokes Solver for Moving and Stationary Grids," AIAA Paper 2005-5039, June 2005.

${ }^{17}$ Vatsa, V., Carpenter, M., and Lockard, D., "Re-evaluation of an Optimized Second Order Backward Difference (BDF2OPT) Scheme for Unsteady Flow Applications," AIAA Paper 2010-0122, January 2010.

${ }^{18}$ Spalart, P. R., "Young Person's Guide to Detached-Eddy Simulation Grids," NASA CR-211032, July 2001.

${ }^{19}$ Spalart, P. R., Deck, S., Shur, M. L., Squires, K. D., Strelets, M. K., and Travin, A., "A New Version of Detached-Eddy Simulation, Resistant to Ambiguous Grid Densities," Theoretical and Computational Fluid Dynamics, Vol. 20, No. 3, 2006, pp. 181-195.
} 
${ }^{20}$ Carlson, J.-R., Vatsa, V. N., and White, J., "Validation of a Node-Centered Wall Function Modles for the Unstructured Flow Code FUN3D," AIAA Paper 2015-xxxx, June 2015.

${ }^{21}$ Spalart, P. R. and Allmaras, S., “A One-Equation Turbulence Model for Aerodynamic Flows,” La Recherche Aerospatiale, Vol. 1, No. 1, 1994, pp. 5-21.

${ }^{22}$ Menter, F. R., “Two-equation Eddy-viscosity Turbulence Models for Engineering Applications,” AIAA Journal, Vol. 32, No. 8, 1994, pp. 15981605.

${ }^{23}$ Knopp, T., Alrutz, T., and Schwamborn, D., "A grid and flow adaptive wall-function method for RANS turbulence modeling," Journal of Computational Physics, Vol. 220, No. 1, 2006, pp. 19-40.

${ }^{24}$ Zawodny, N., Liu, F., Yardibi, T., Cattafesta, L., Khorrami, M., Neuhart, D., and Van de Ven, T., "A Comparative Study of a 1/4-scale Gulfstream G550 Aircraft Nose Gear Model,” AIAA Paper 2009-3153, May 2009.

${ }^{25} 3 r d$ AIAA Workshop on Benchmark Problems for Airframe Noise Computations Problems. AIAA, 2014, Atlanta, Georgia, June 14-15, 2014.

${ }^{26}$ Carlson, J.-R., "Inflow/Outflow Boundary Conditions with Application to FUN3D," NASA TM 2011-217181, NASA Langley Research Center, Oct. 2011

${ }^{27}$ Neuhart, D., Khorrami, M., and Choudhari, M., “Aerodynamics of a Gulfstream G550 Nose Landing Gear Model," AIAA Paper 2009-3152, May 2009.

${ }^{28}$ Ffowcs Williams, J. E. and Hawkings, D. L., "Sound Generated by Turbulence and Surfaces in Arbitrary Motion," Philosophical Transactions of the Royal Society, Vol. A264, No. 1151, 1969, pp. 321-342.

${ }^{29}$ Brentner, K. S., Lopes, L. V., Chen, H. N., and Horn, J. F., "Near Real-Time Simulation of Rotorcraft Acoustics and Flight Dynamics," 59th Annual Forum, AHS International, Alexandria, VA, 2003.

${ }^{30}$ Bres, G. A., Brentner, K. S., Perez, G., and Jones, H. E., "Maneuvering Rotorcraft Noise Prediction,” Journal of Sound and Vibration, Vol. 275, No. 3-5, 2004, pp. 719-738.

${ }^{31}$ AVEC Web page: http://www.avec-engineering.com/products.html, March 2015.

${ }^{32}$ Sijtsma, P., "CLEAN Based on Spatial Source Coherence," AIAA Paper 2007-3436, 2007. 


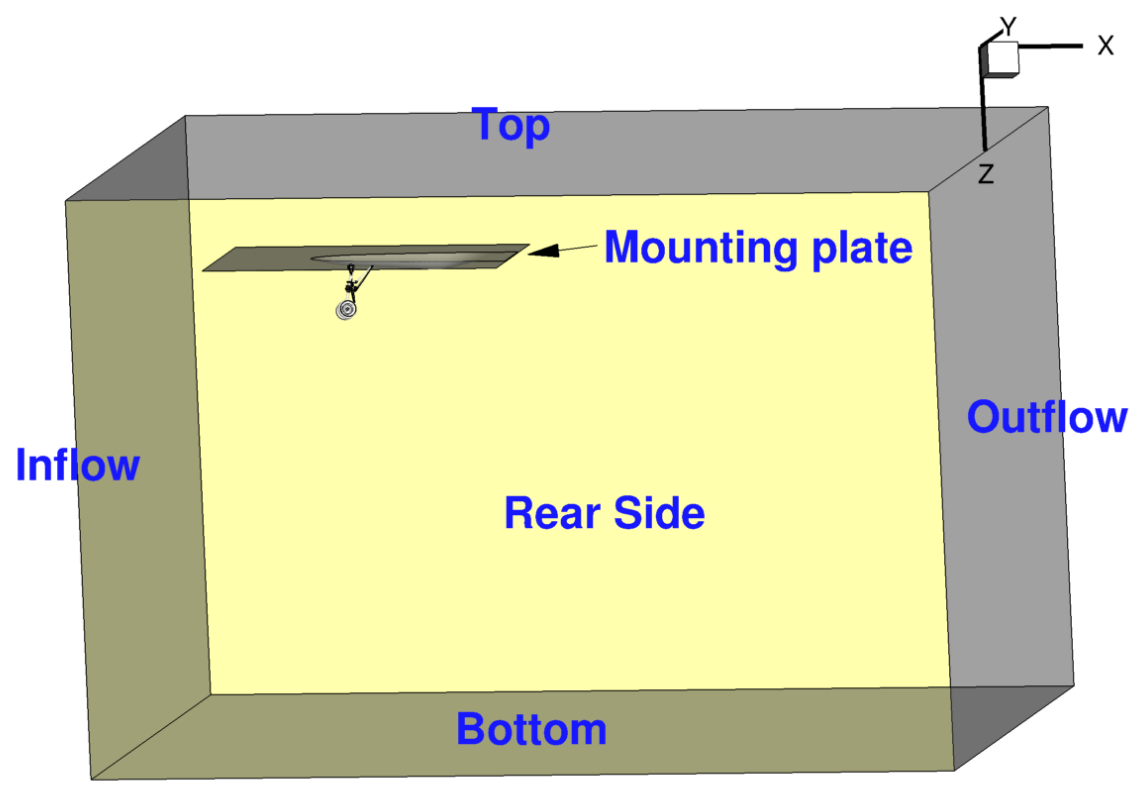

Figure 1. Computational model of the nose landing gear in UFAFF tunnel.

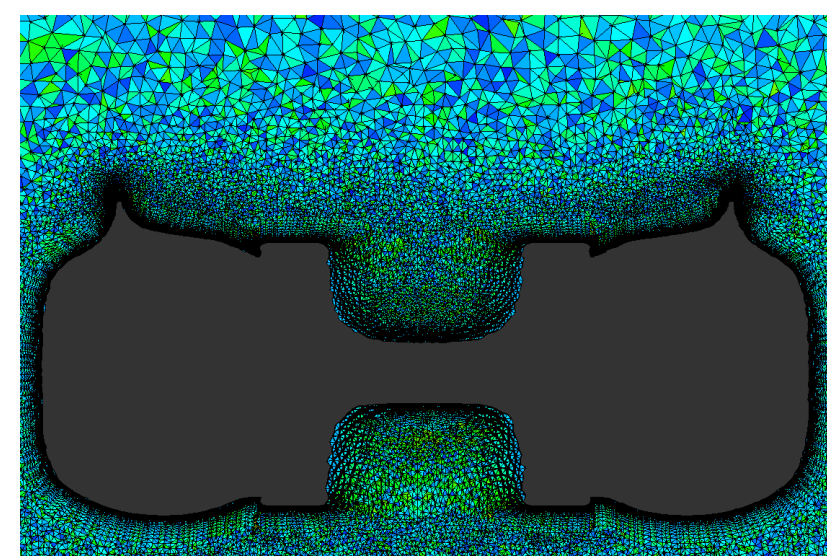

(a) Grid with no baffle

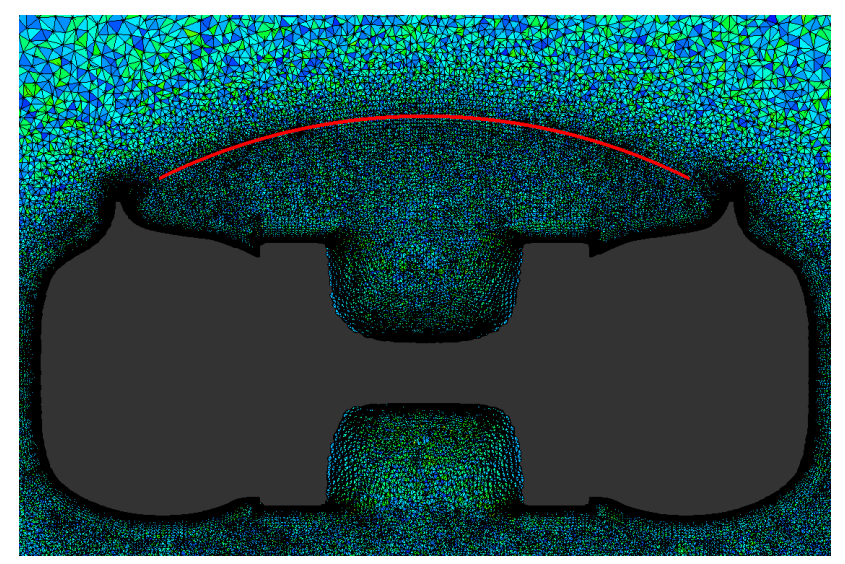

(b) Grid with baffle

Figure 2. Comparison of mesh distribution at wheel cavity with and without baffle. 


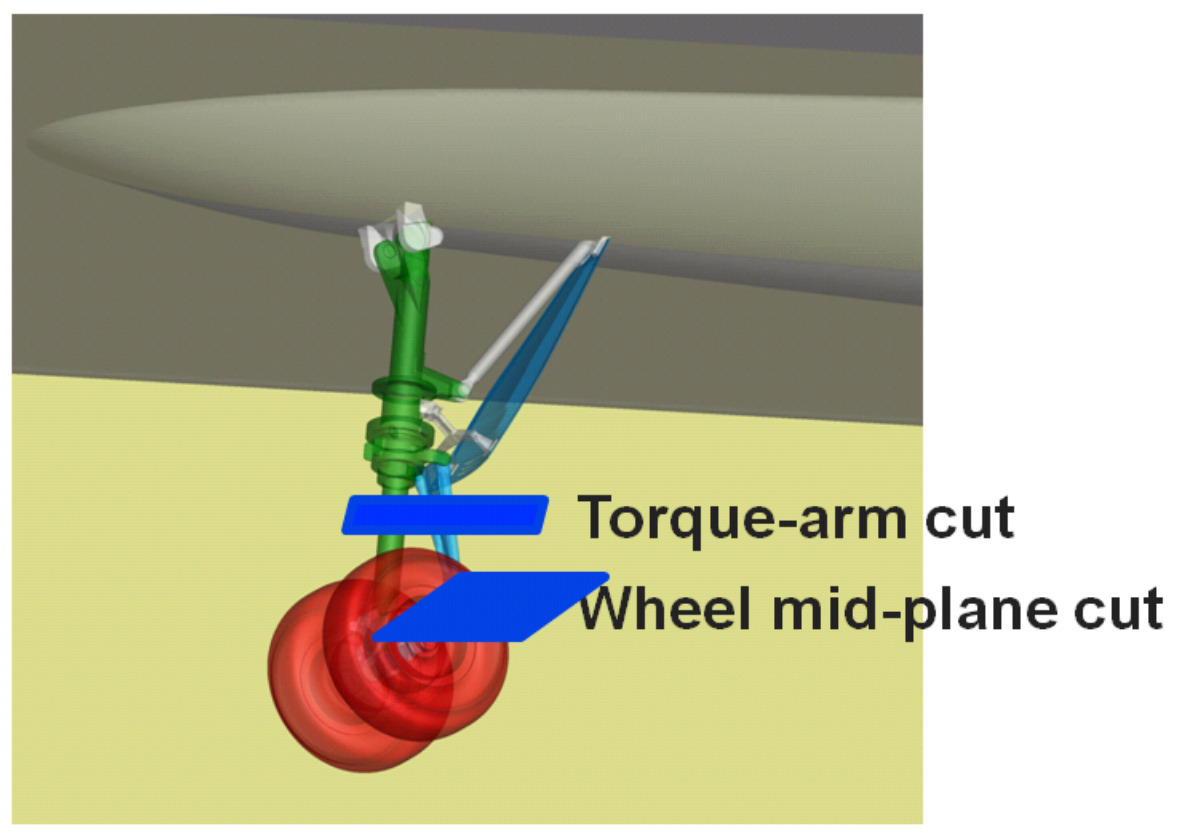

Figure 3. Wheel and torque-arm cut locations.

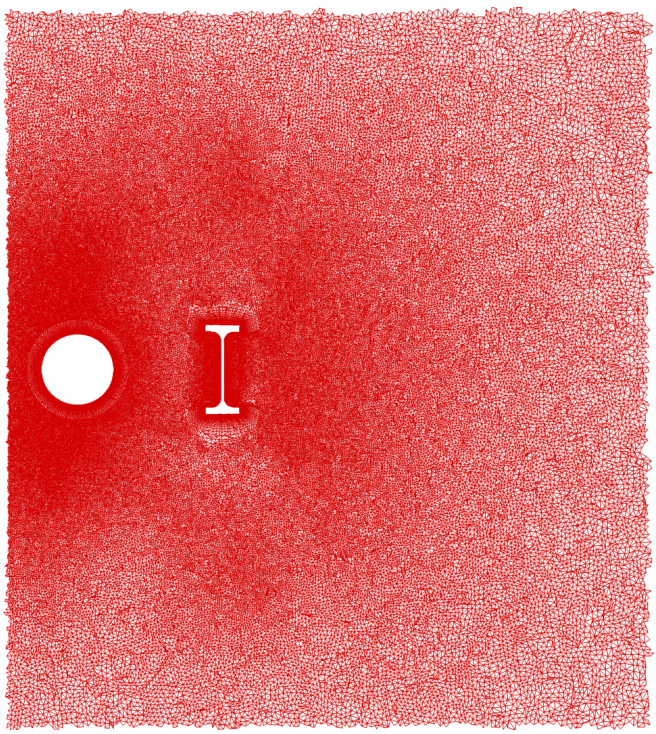

(a) Pointwise grid: 62M $\left(y^{+}=1\right)$

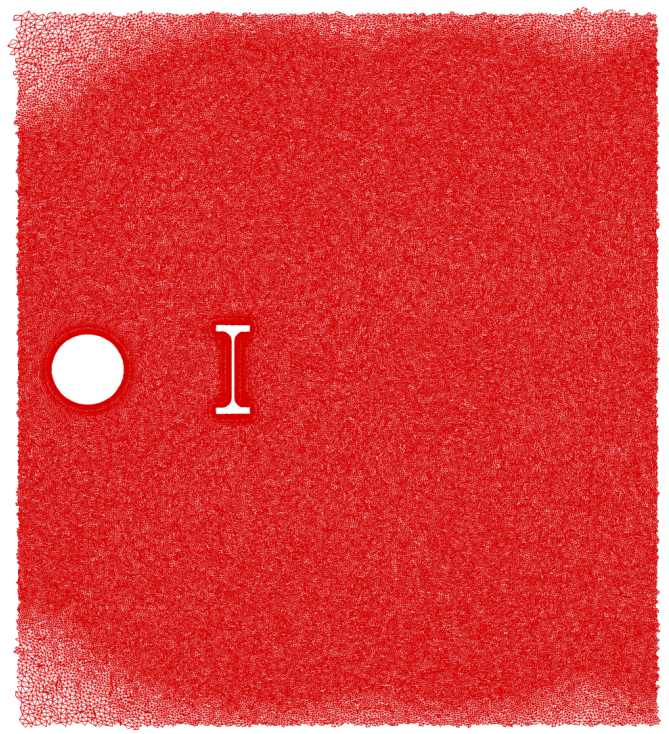

(b) Reference grid: 104M

Figure 4. Comparison of Pointwise grid with reference grid at torque-arm cut. 


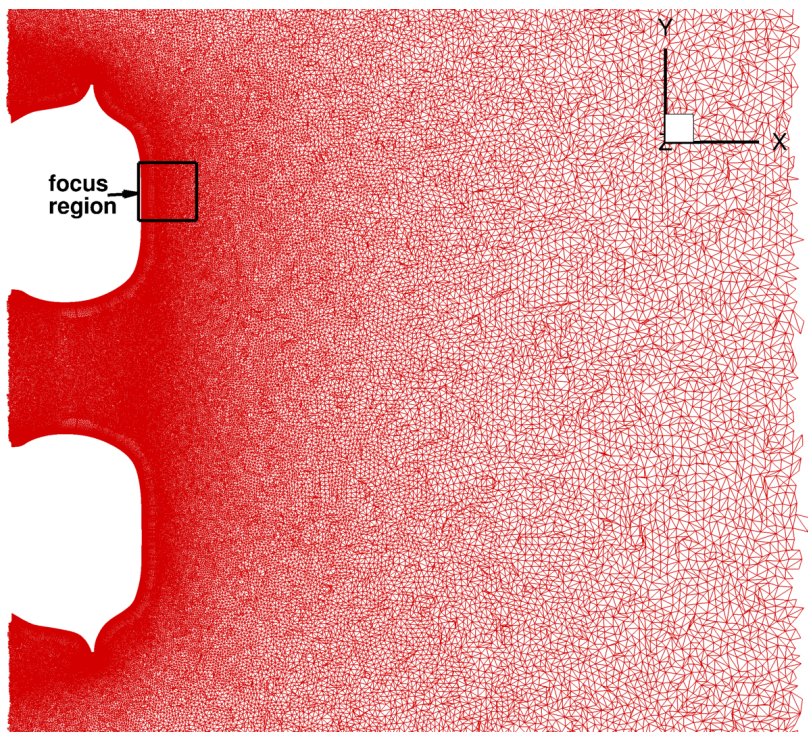

(a) Pointwise grid: 62M $\left(y^{+}=1\right)$

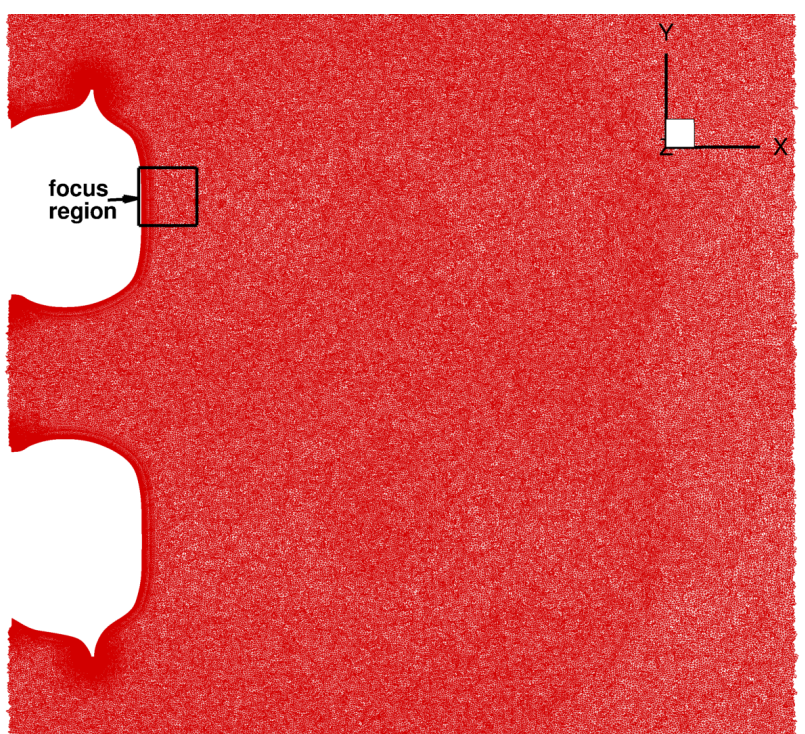

(b) Reference grid: 104M

Figure 5. Comparison of mesh distribution at mid-section of tire wake, global view.

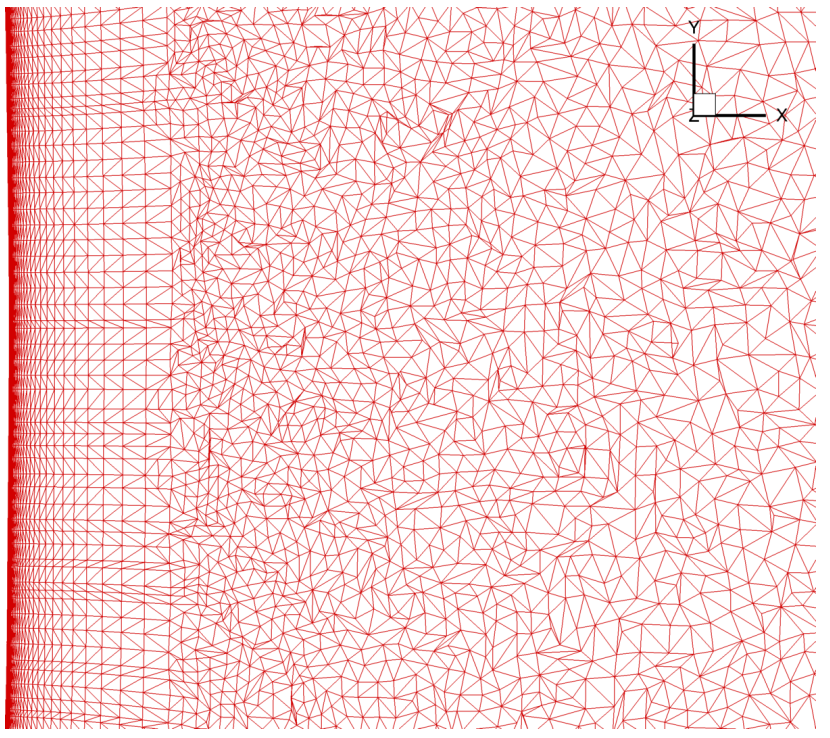

(a) Pointwise grid: 62M $\left(y^{+}=1\right)$

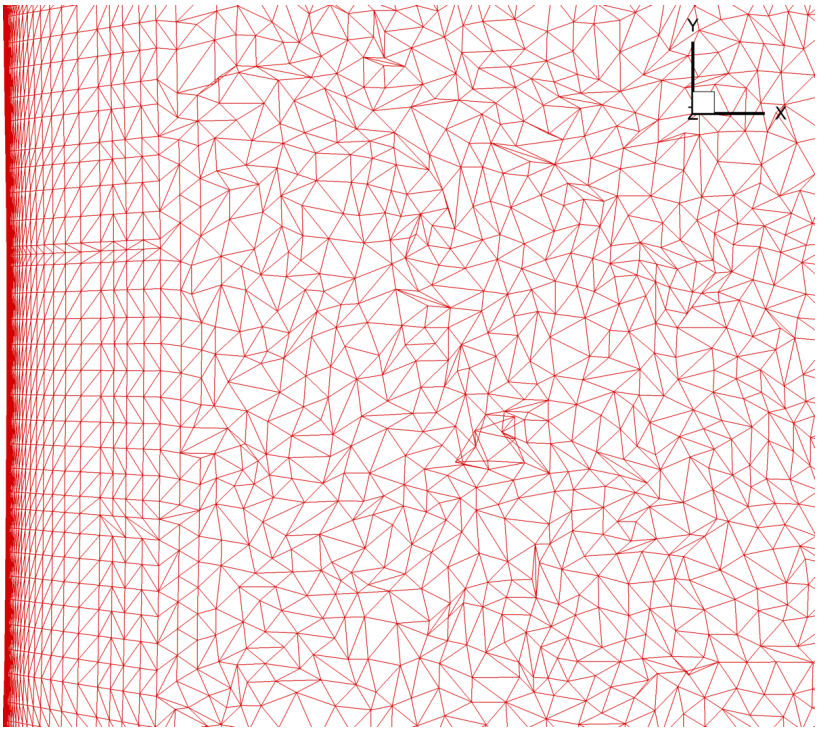

(b) Reference grid: 104M

Figure 6. Comparison of mesh distribution at mid-section of tire wake, zoomed view of focus region identified in Fig. 5. 


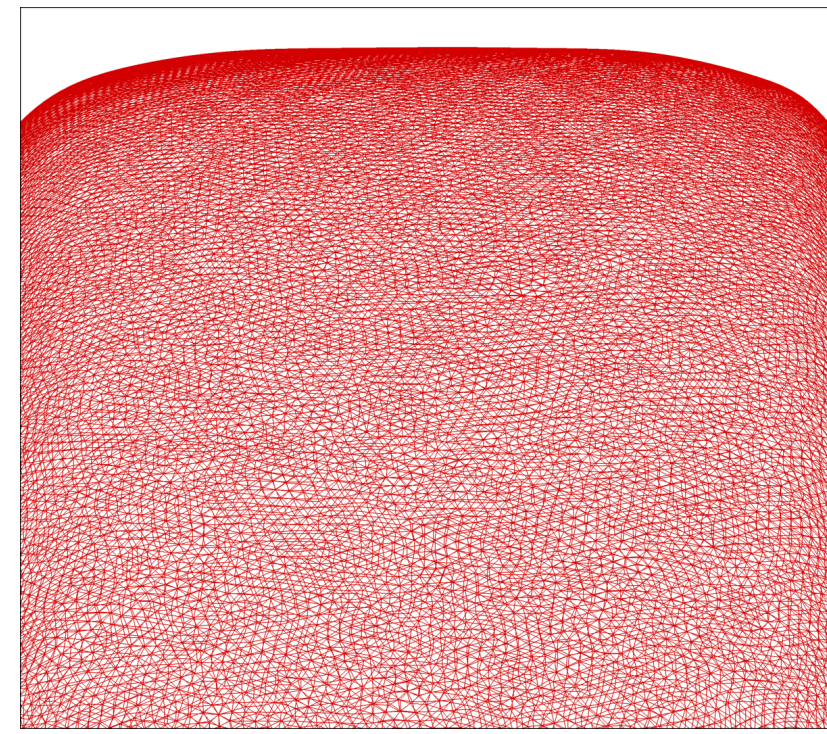

(a) Pointwise grid: 62M $\left(y^{+}=1\right)$

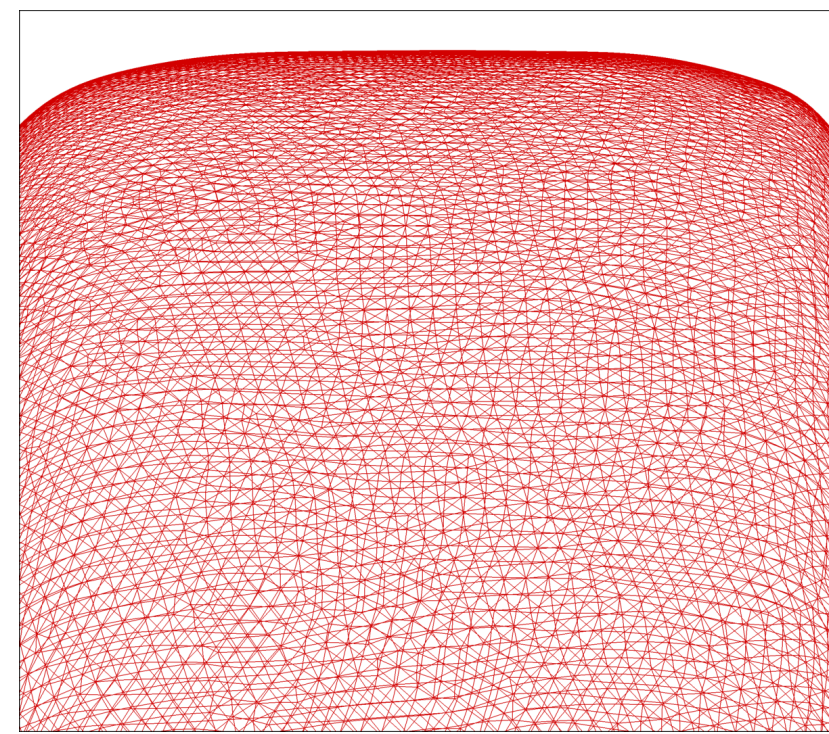

(b) Reference grid: 104M

Figure 7. Comparison of surface mesh distribution at mid-section of port wheel



Figure 8. Surface pressure distributions on port wheel. 


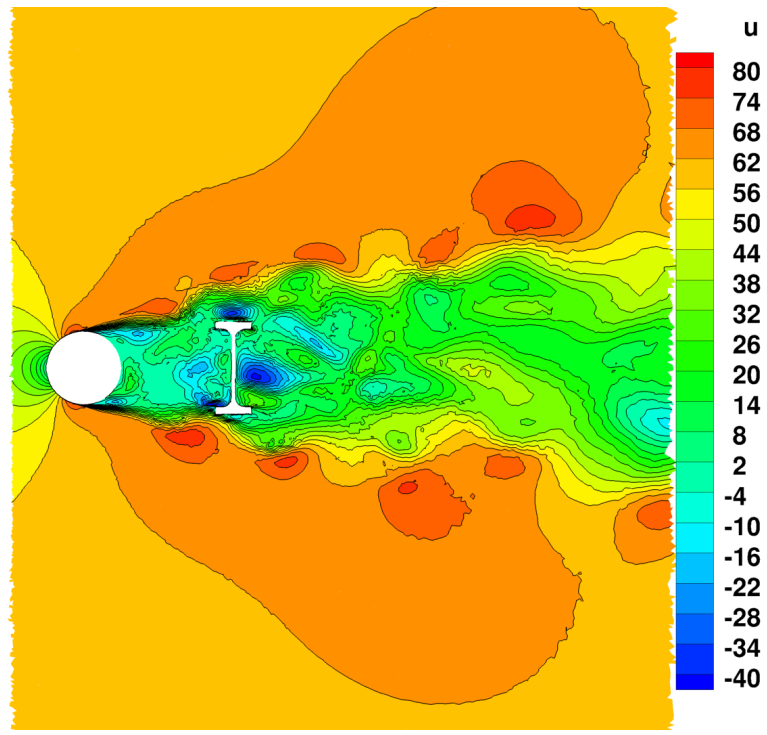

(a) Pointwise grid: 62M $\left(y^{+}=1\right)$

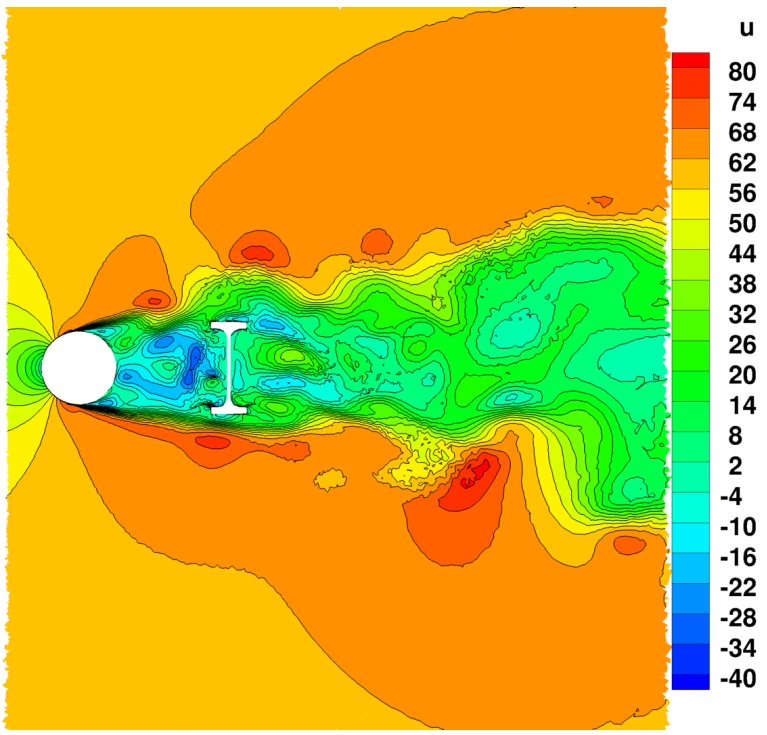

(b) Pointwise grid: 45M $\left(y^{+}=25\right)$



(c) Reference grid: 104M

Figure 9. Comparison of instantaneous streamwise velocity contours at torque-arm cut. 


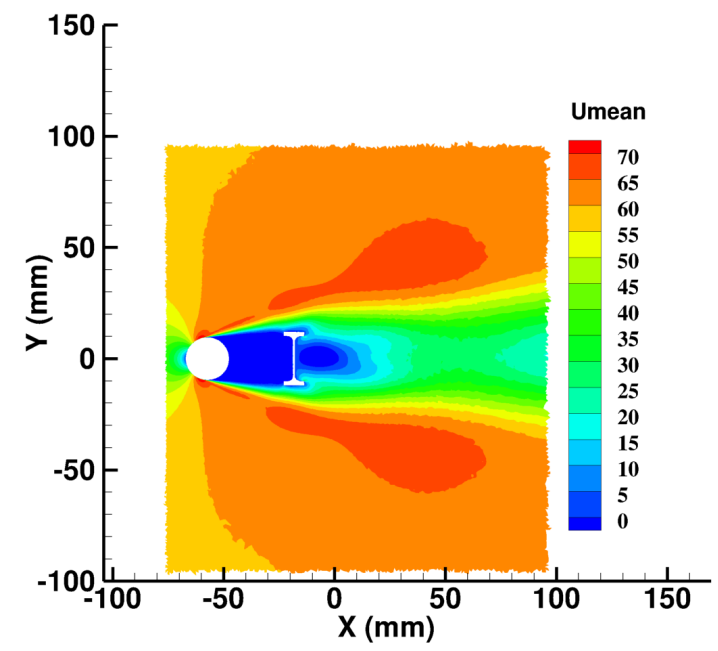

(a) Pointwise grid: 62M $\left(y^{+}=1\right)$

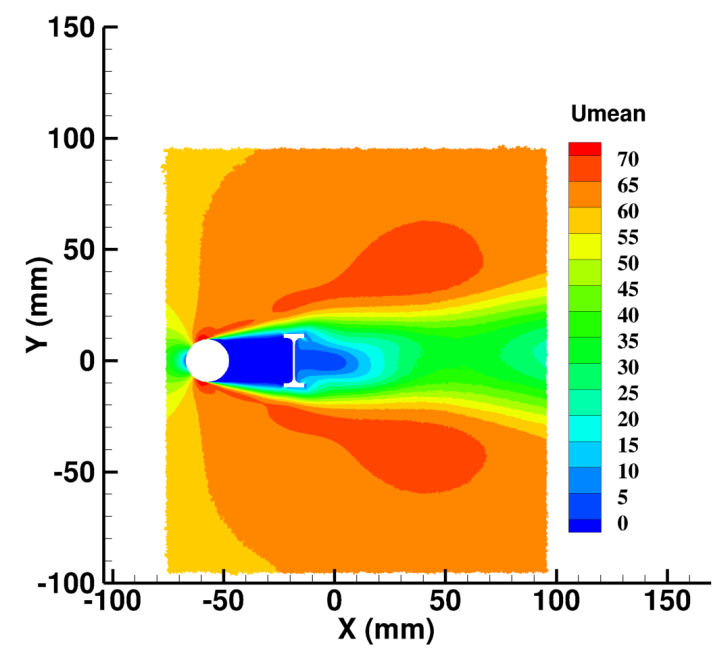

(c) Reference grid: 104M

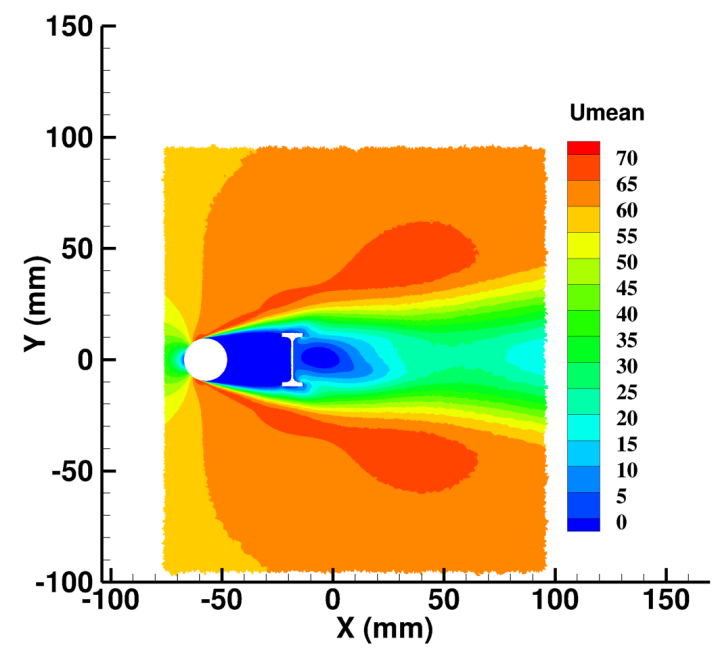

(b) Pointwise grid: 45M $\left(y^{+}=25\right)$

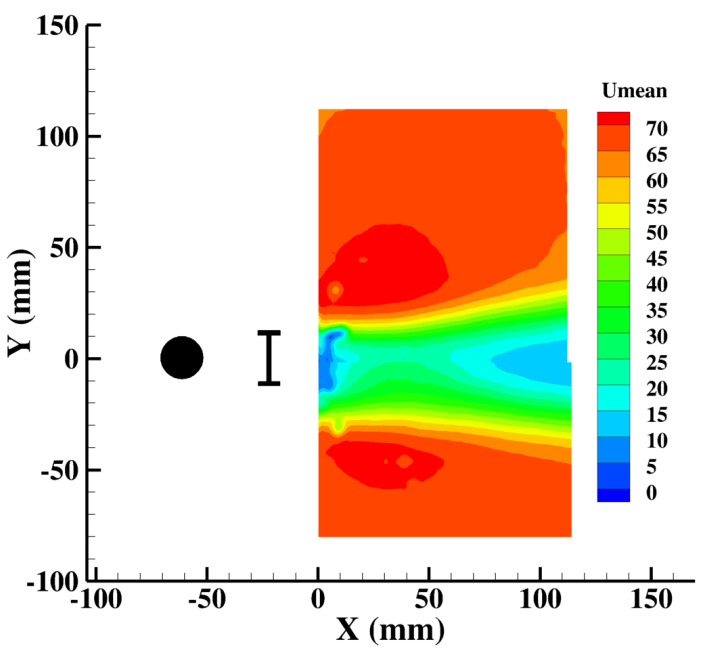

(d) Exp. PIV data

Figure 10. Comparison of time-averaged streamwise velocity contours at torque-arm cut. 


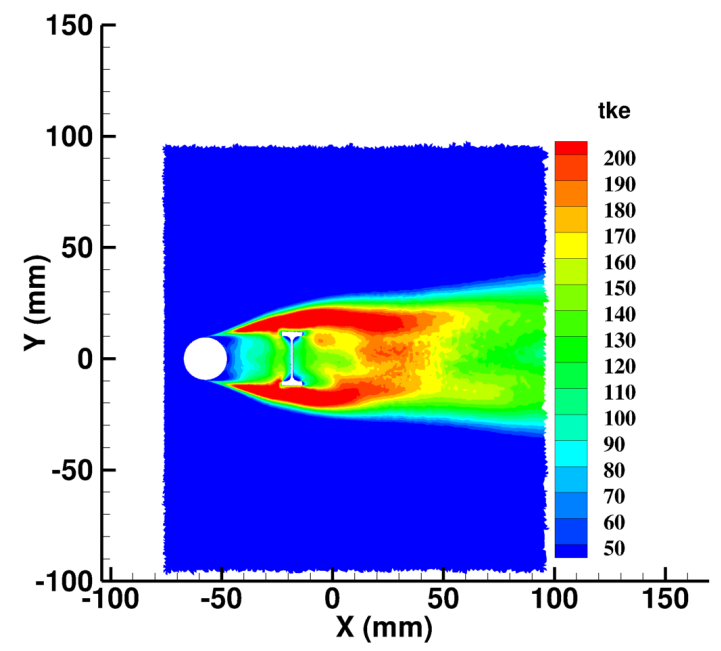

(a) Pointwise grid: 62M $\left(y^{+}=1\right)$



(c) Reference grid: 104M

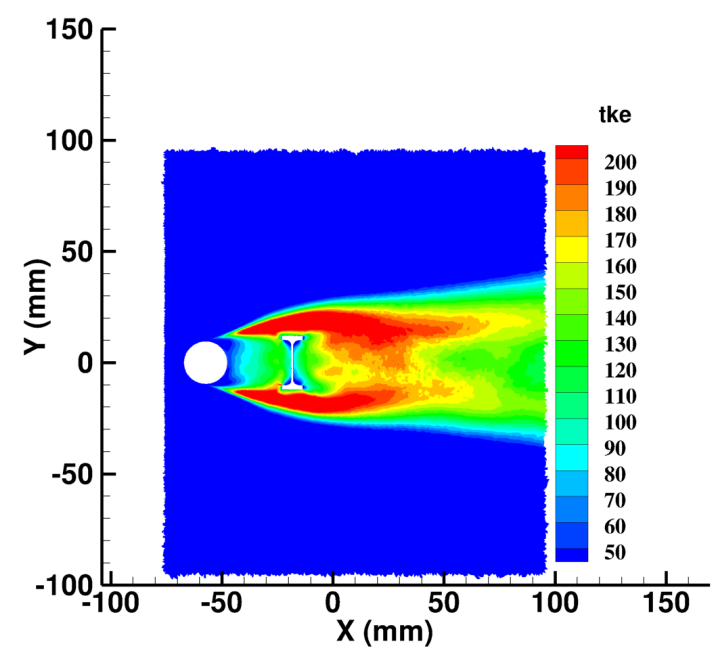

(b) Pointwise grid: 45M $\left(y^{+}=25\right)$



(d) Exp. PIV data

Figure 11. Comparison of 2-D turbulence kinetic energy contours at torque-arm cut. 


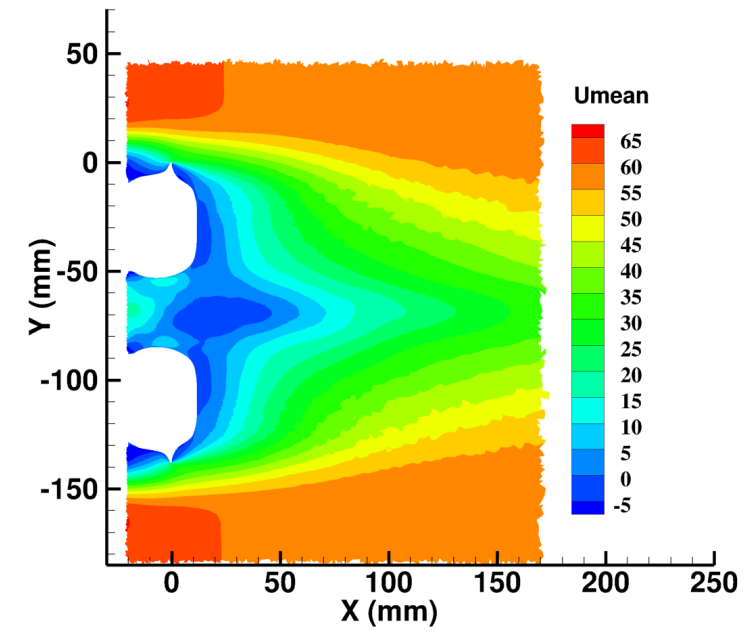

(a) FUN3D simulation, PW-62M $\left(y^{+}=1\right)$

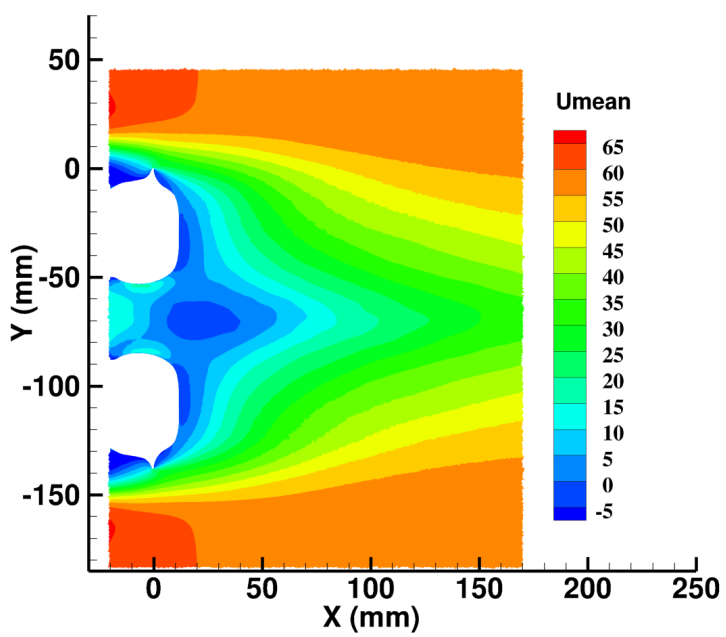

(c) FUN3D simulation, 104M ref. grid

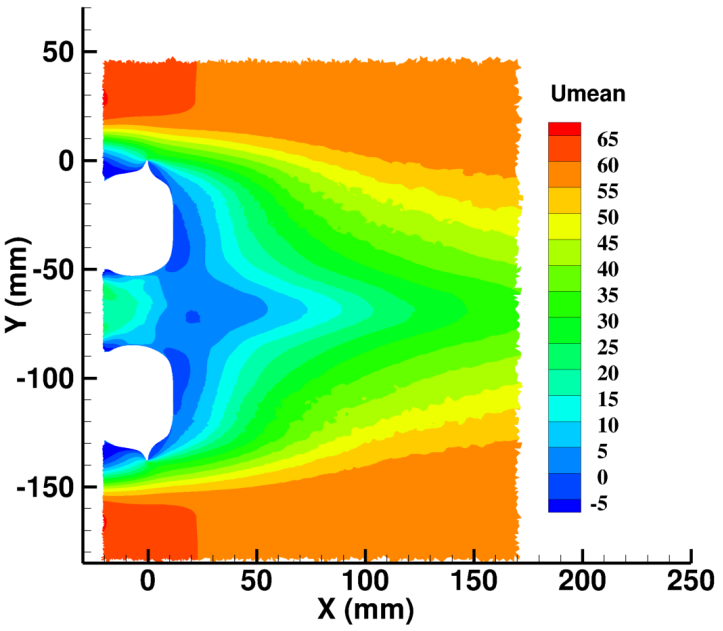

(b) FUN3D simulation, PW-45M $\left(y^{+}=25\right)$

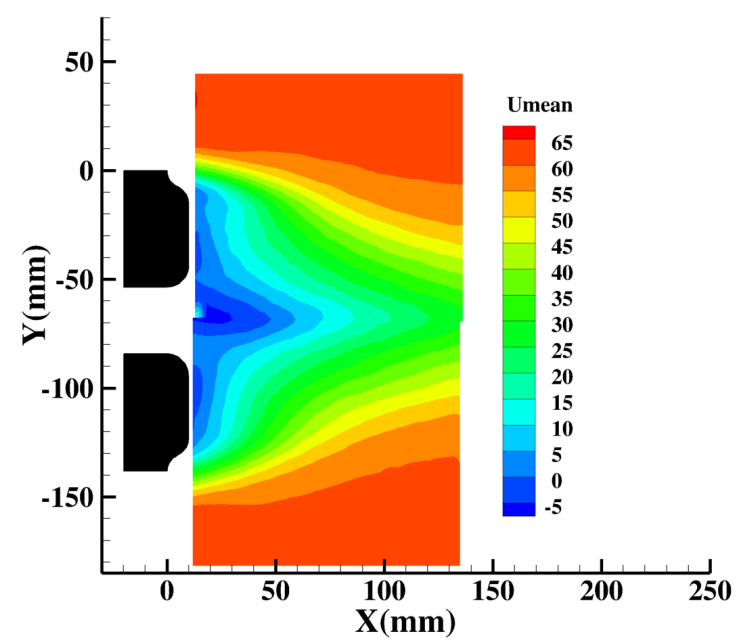

(d) Exp. PIV data

Figure 12. Comparison of time-averaged streamwise velocity contours, mid-wheel plane cut. 


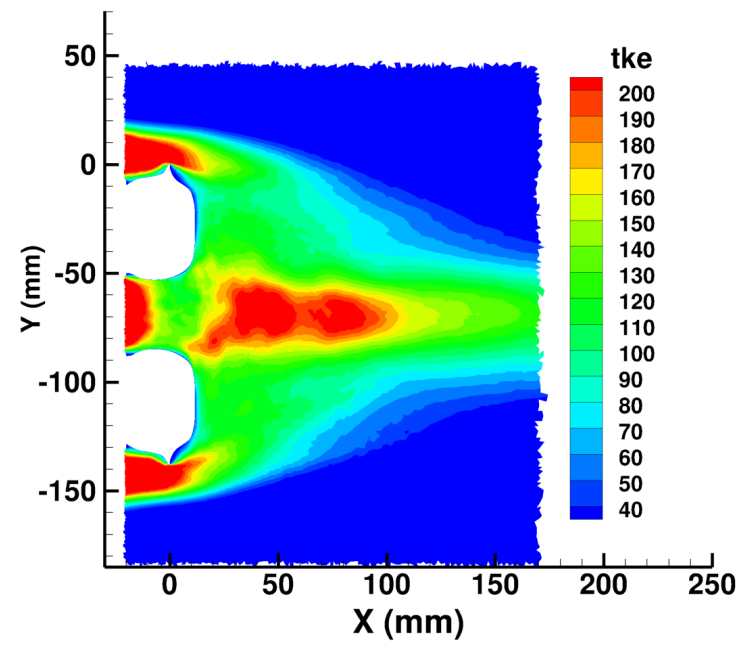

(a) FUN3D simulation, PW-62M $\left(y^{+}=1\right)$

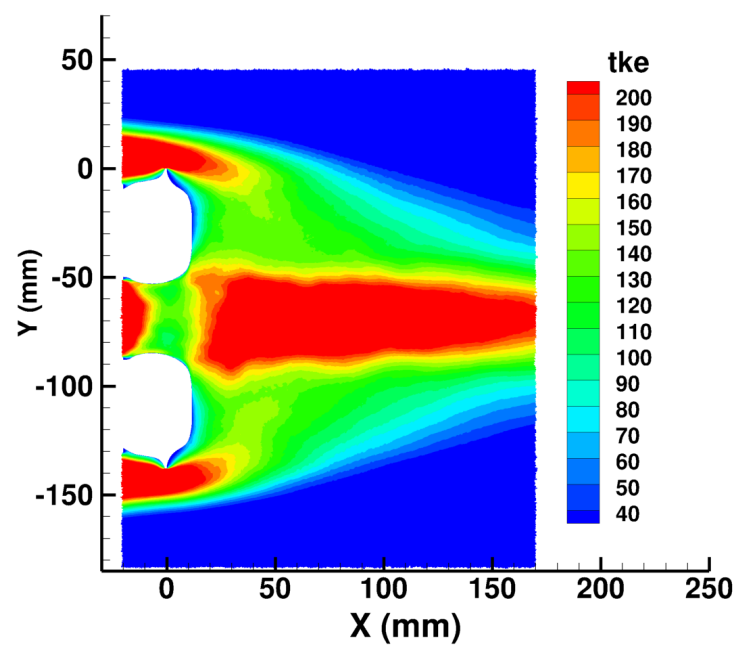

(c) FUN3D simulation, 104M ref. grid



(b) FUN3D simulation, PW-45M $\left(y^{+}=25\right)$



(d) Exp. PIV data

Figure 13. Comparison of 2-D turbulence kinetic energy contours, mid-wheel plane cut. 


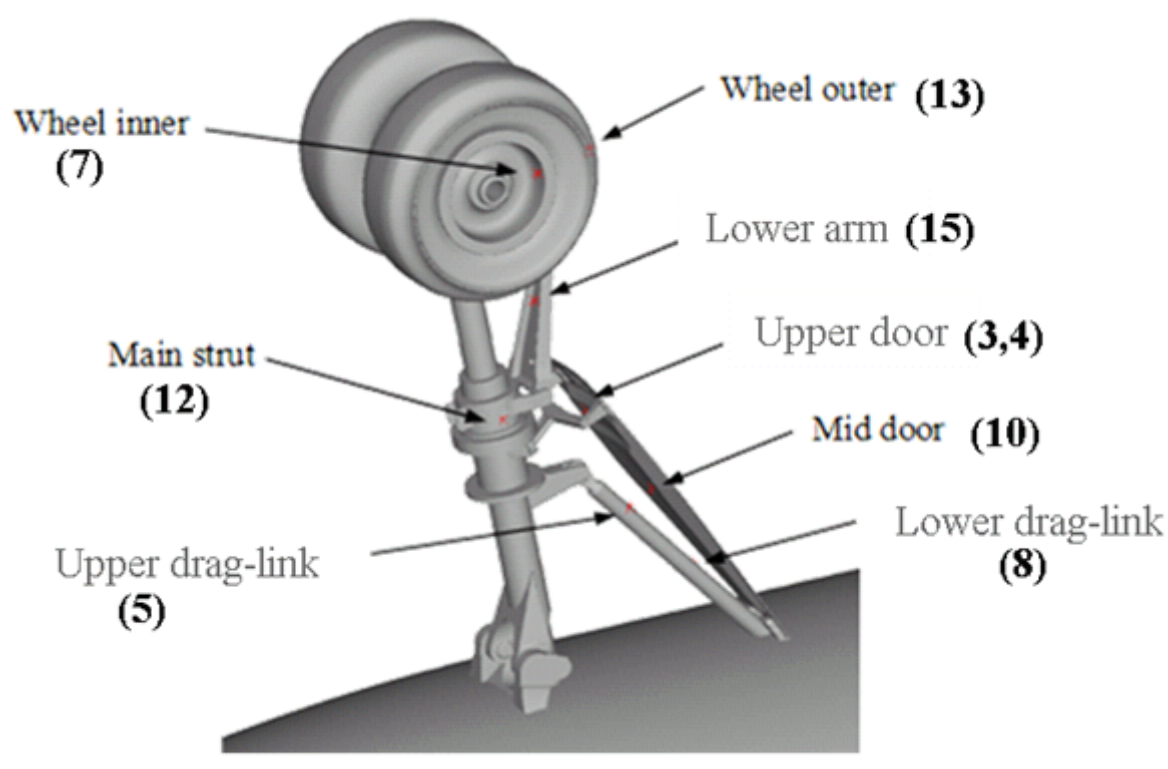

Figure 14. Surface mounted Kulite ${ }^{\circledR}$ sensor locations.

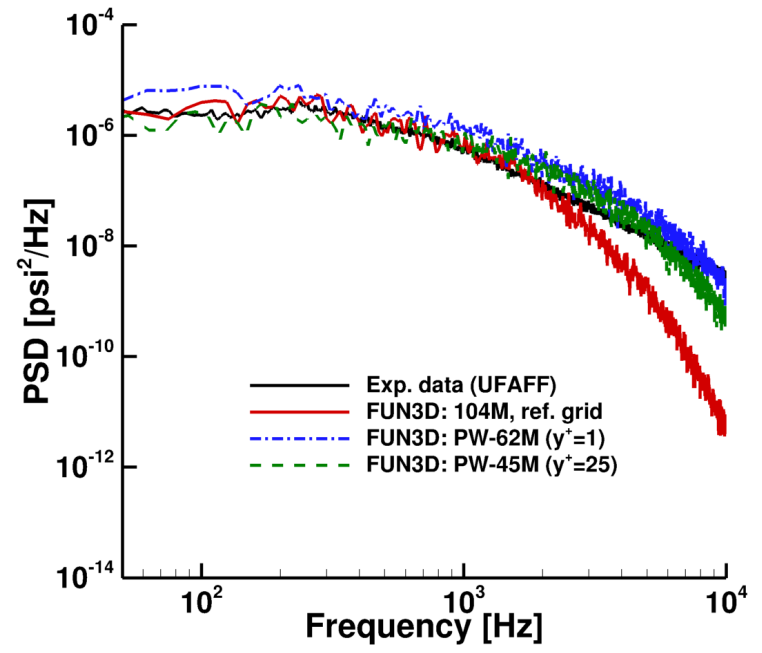

(a) Upper-door, ch. 3

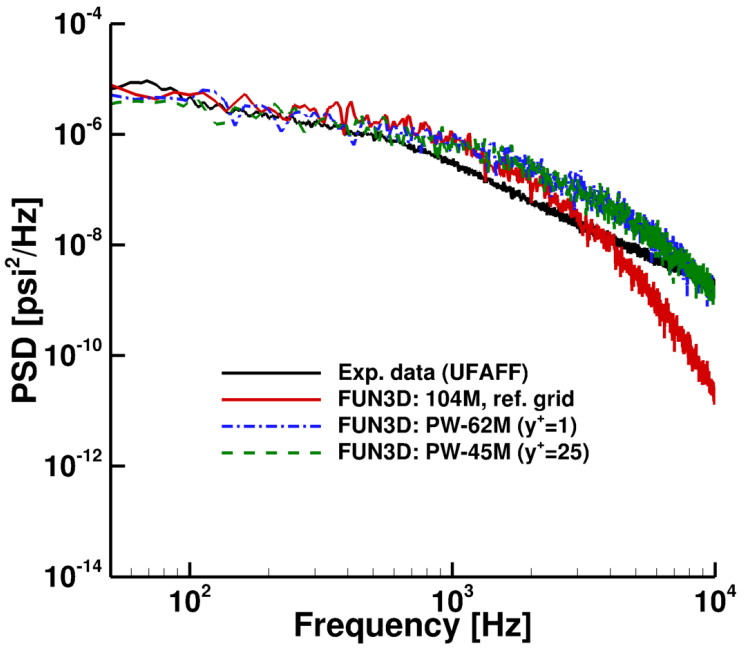

(b) Mid-door, ch. 10

Figure 15. PSD comparisons for Kulite ${ }^{\circledR}$ sensors 3 and 10. 


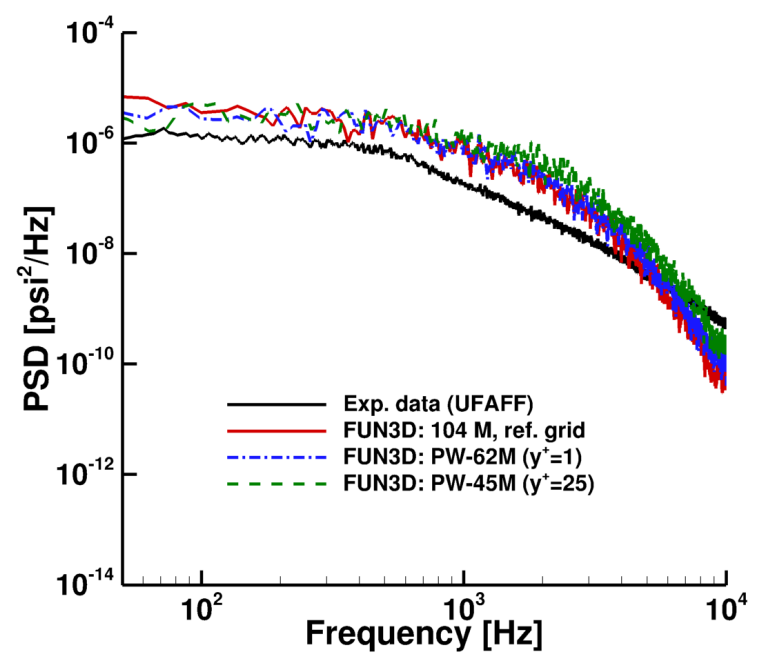

(a) Wheel-inner, ch. 7



(b) Lower arm, ch. 15

Figure 16. PSD comparisons for Kulite ${ }^{\circledR}$ sensors 7 and 15.
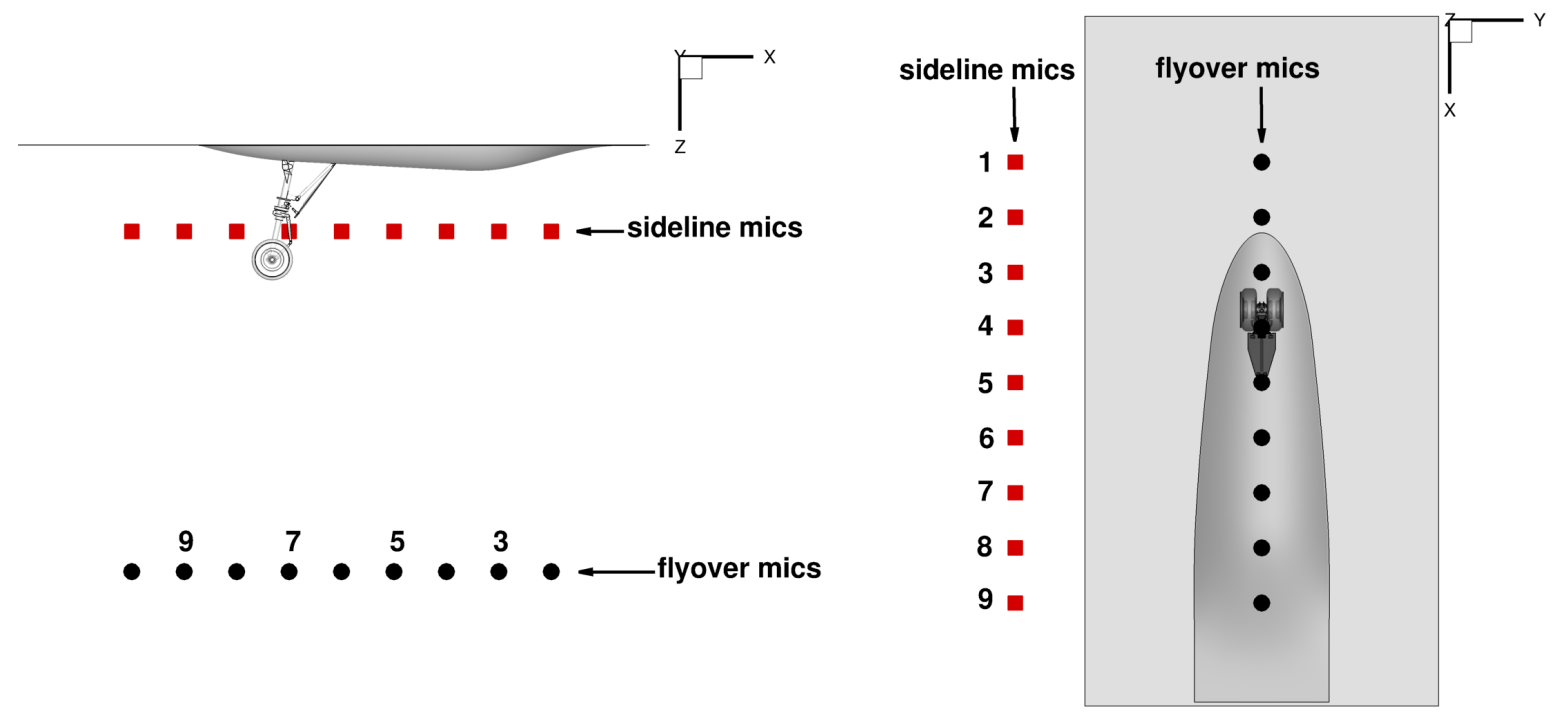

(a) side view

(b) top view

Figure 17. Linear microphone array arrangement in UFAFF. 


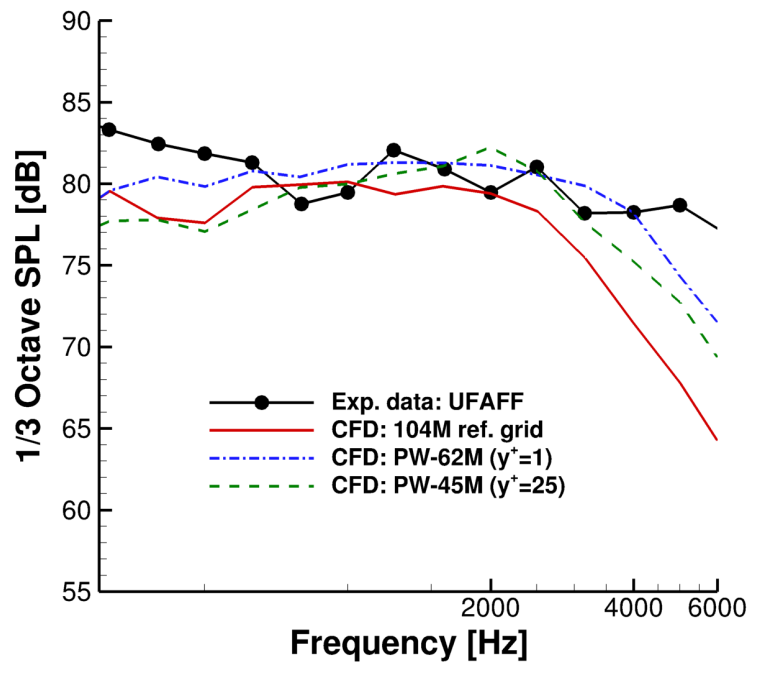

(a) Microphone 4



(b) Microphone 7

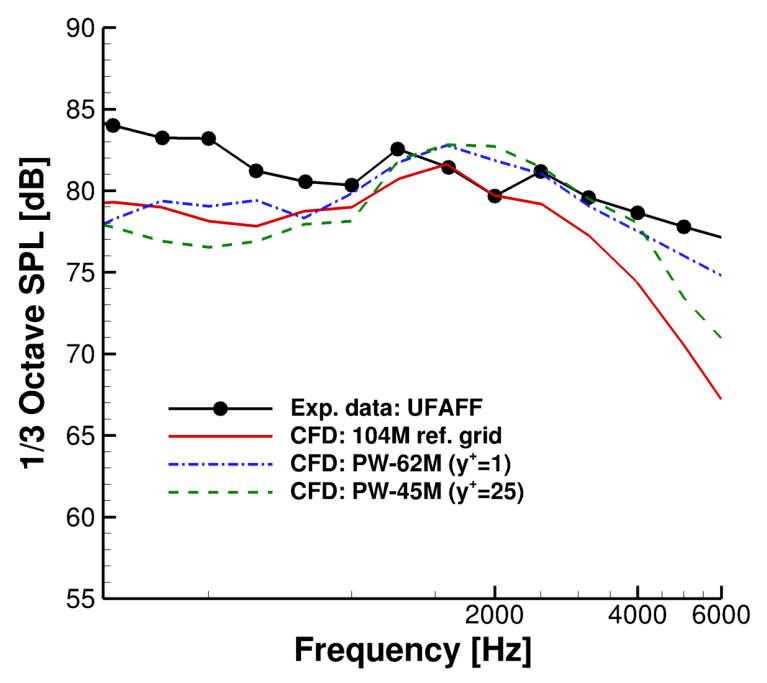

(c) Microphone 9

Figure 18. Sound pressure level comparisons at flyover microphones using solid surface perturbations in the FW-H Solver. 


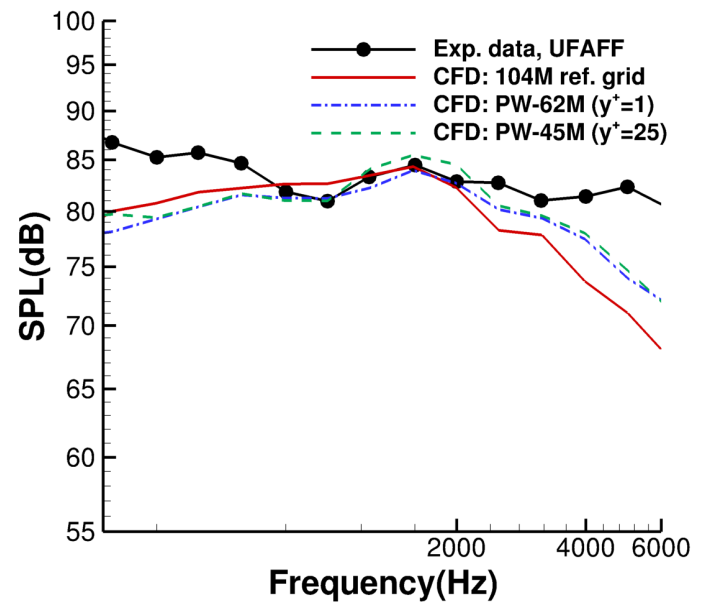

(a) Microphone 3

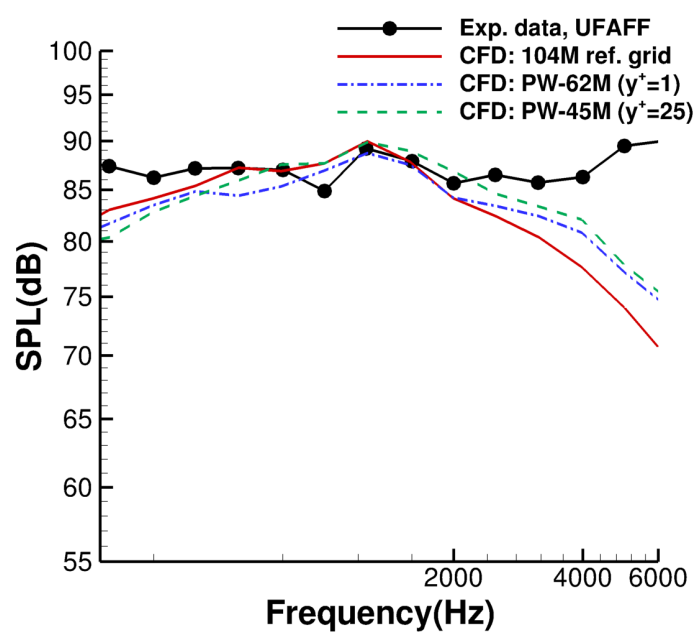

(c) Microphone 7

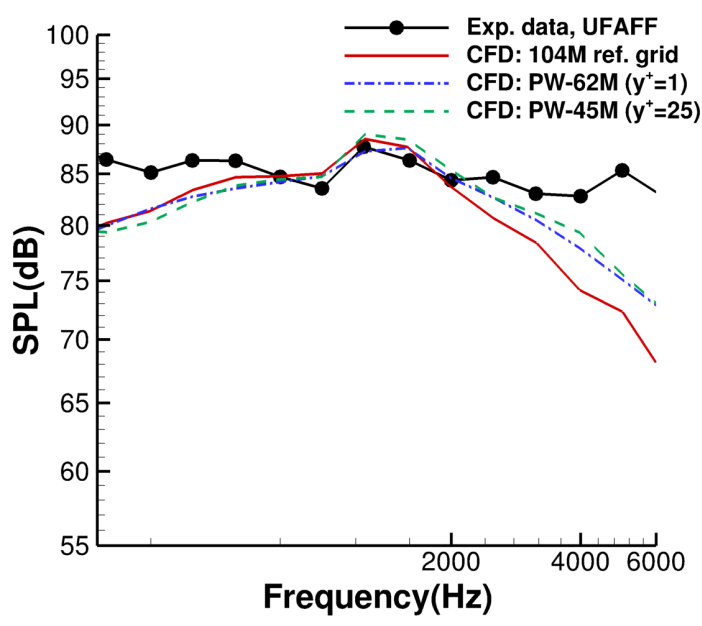

(b) Microphone 5

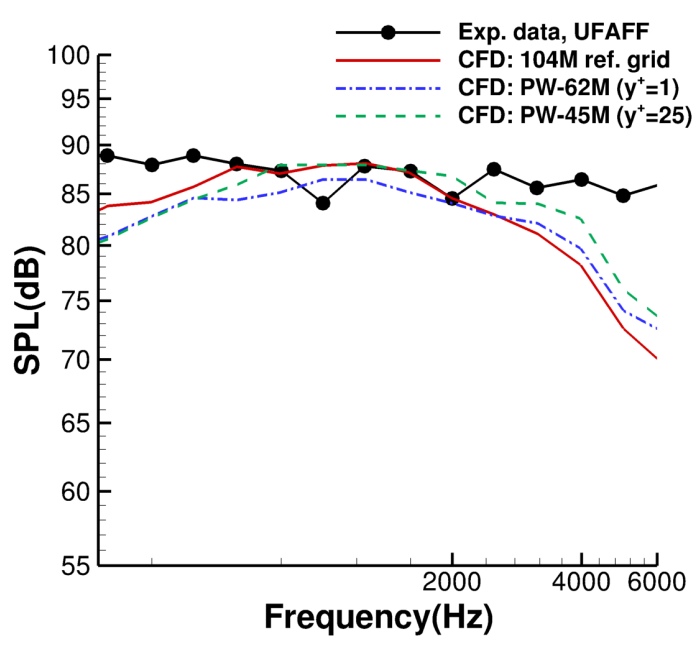

(d) Microphone 9

Figure 19. Sound pressure level comparisons at sideline microphones using solid surface perturbations in the FW-H Solver. 


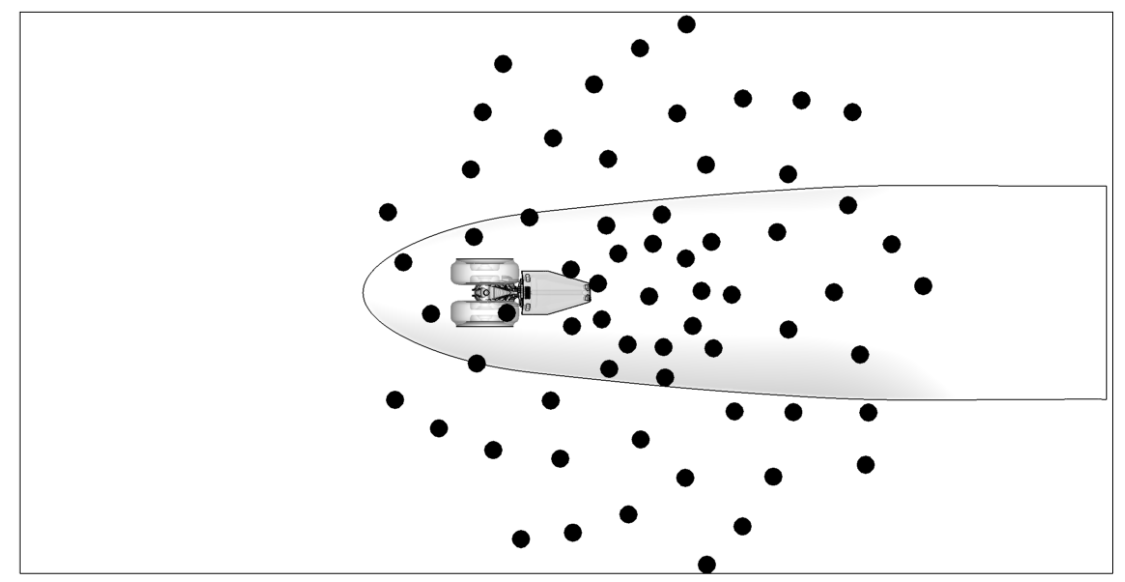

(a) flyover array

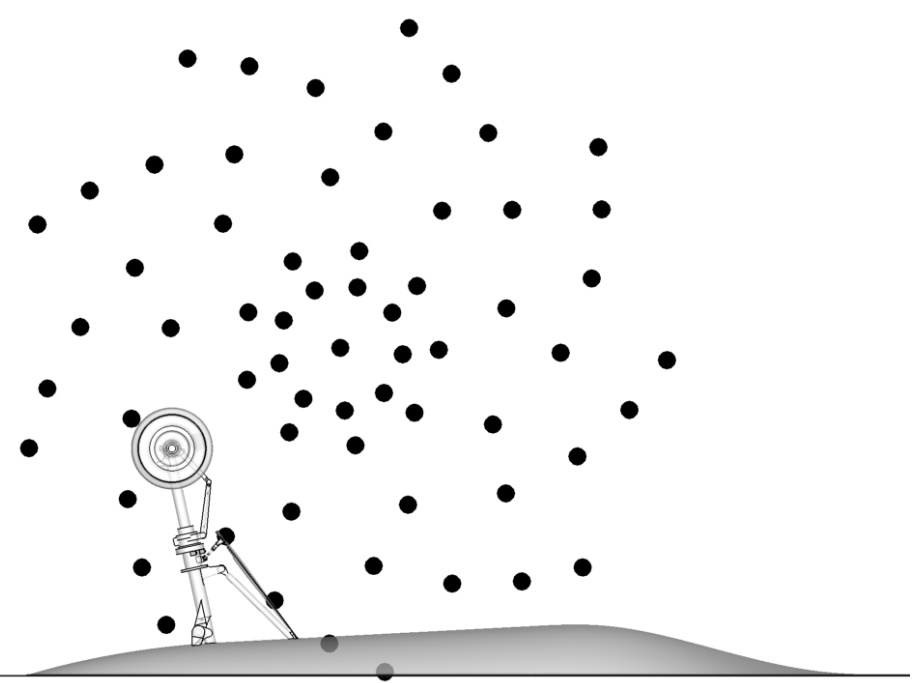

(b) sideline array

Figure 20. Linear acoustic array arrangement in UFAFF. 

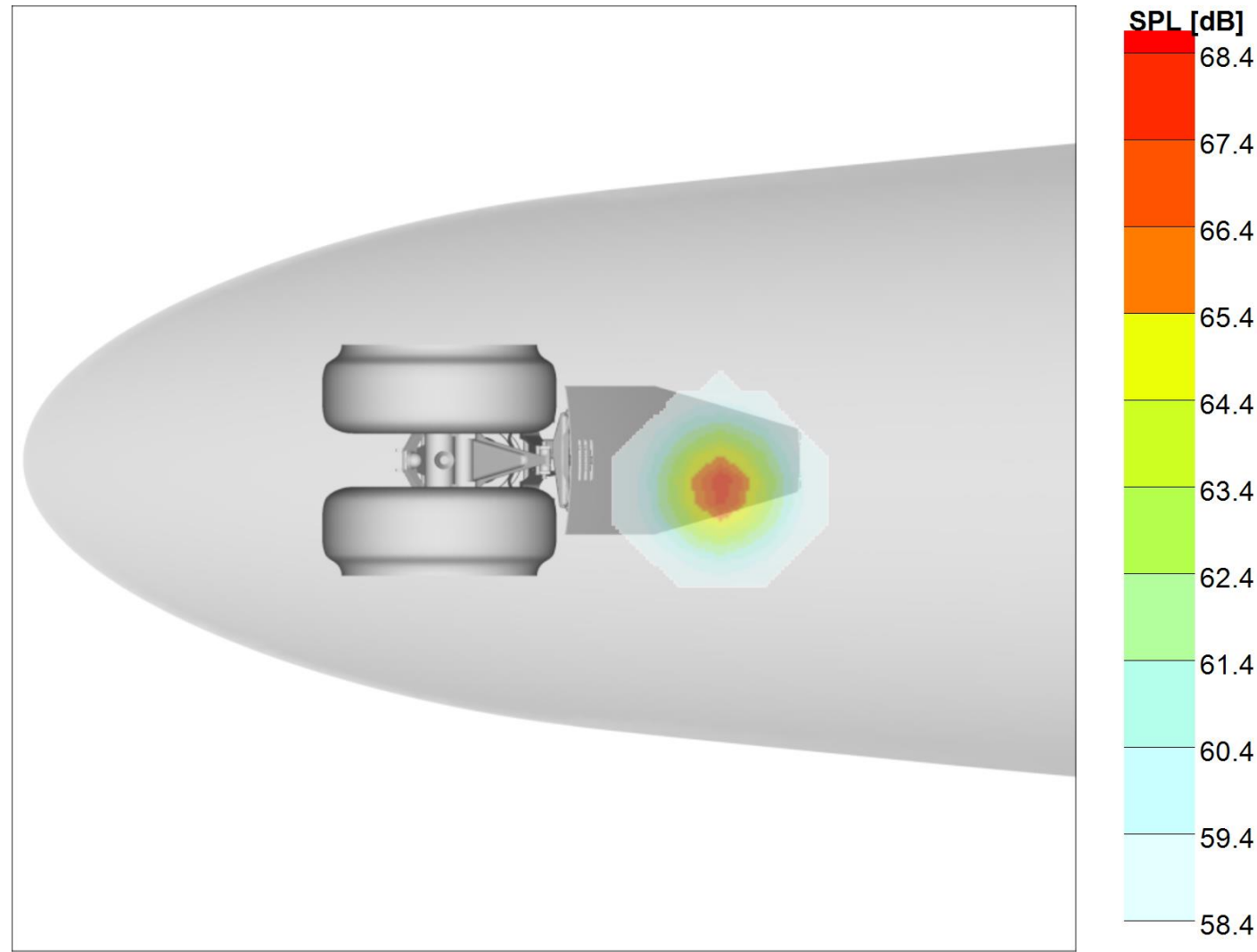

(a) PW-62M



70.3

69.3

68.3

67.3

66.3

65.3

64.3

63.3

62.3

61.3

(b) VGRID 104M

Figure 21. Comparison of flyover noise contour maps at $1 \mathrm{kHz}$. 

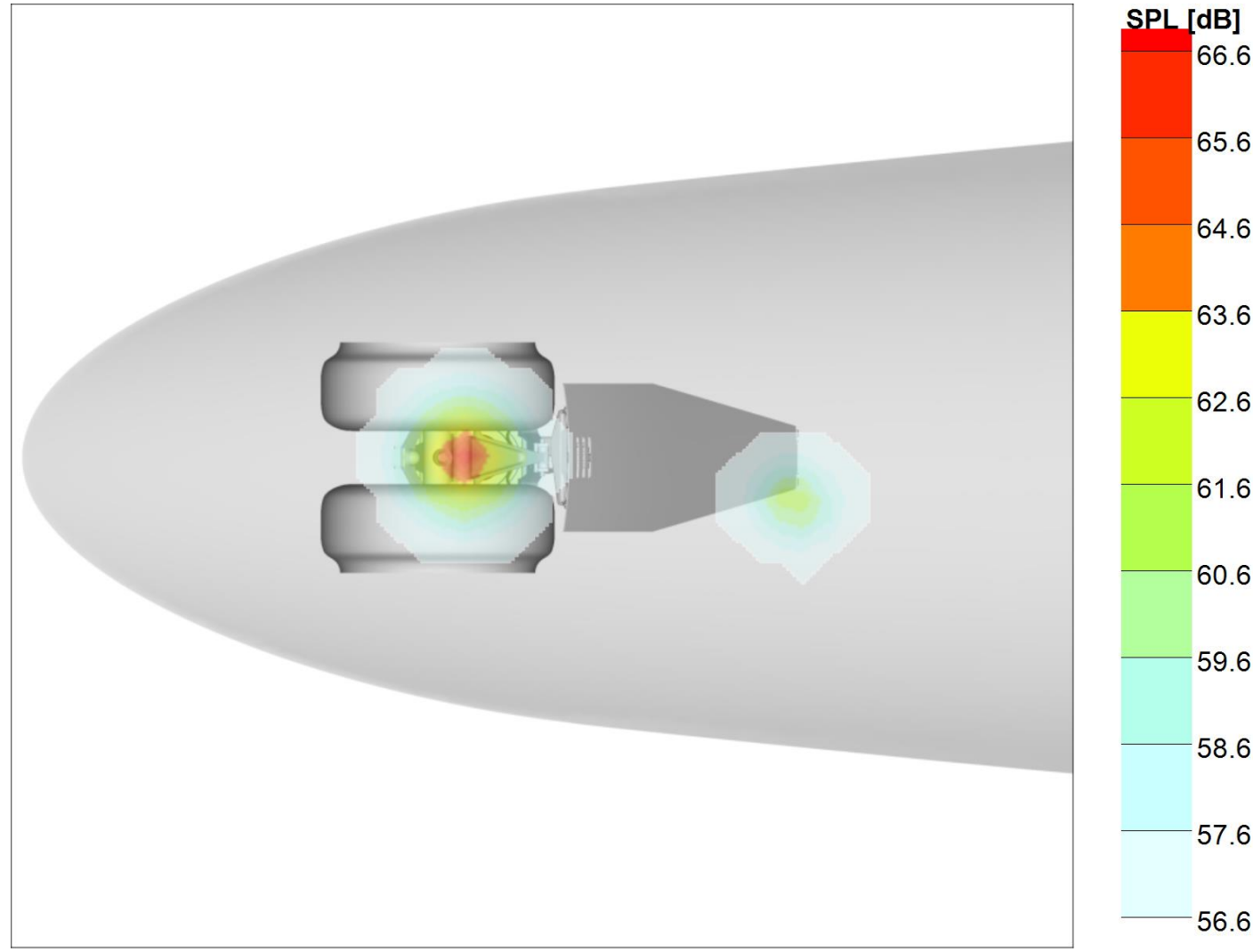

(a) PW-62M

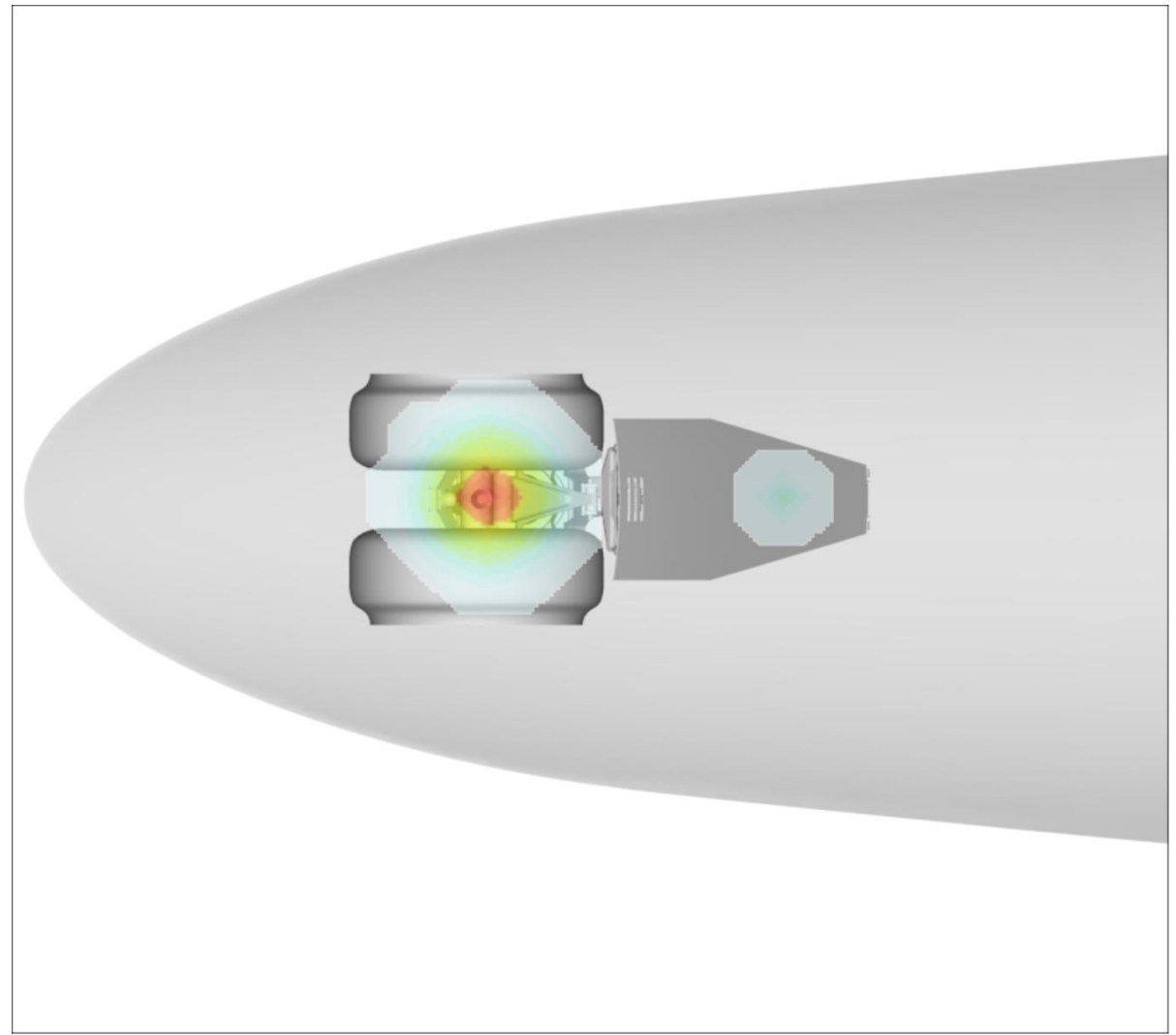

(b) VGRID 104M

Figure 22. Comparison of flyover noise contour maps at $3 \mathrm{kHz}$. 



(a) PW-62M

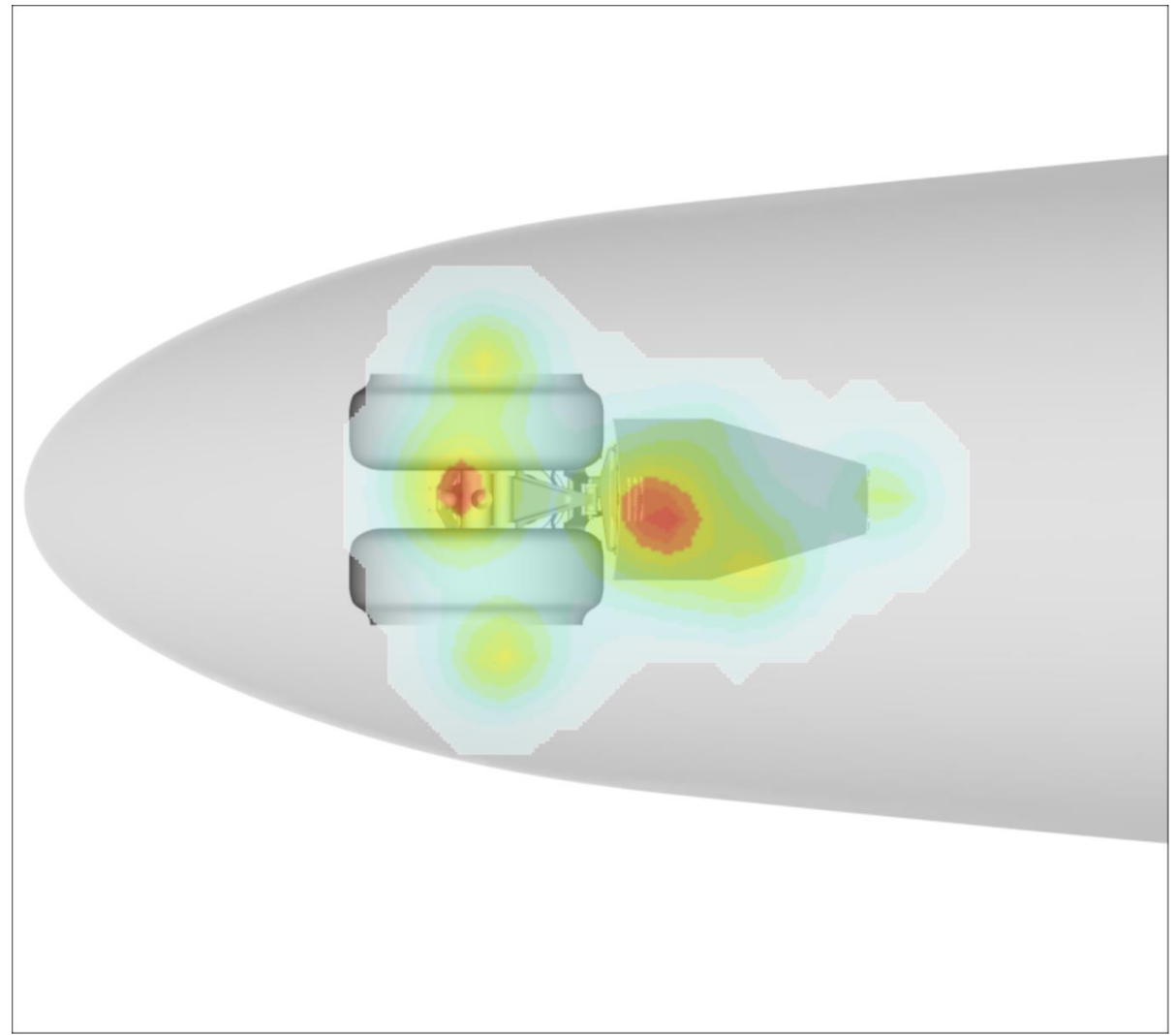

(b) VGRID 104M

Figure 23. Comparison of flyover noise contour maps at $5 \mathrm{kHz}$. 